\title{
On the Gorenstein locus of the punctual Hilbert scheme of degree 11 ts
}

\author{
Gianfranco Casnati $^{\mathrm{a}, *}$, Roberto Notari ${ }^{\mathrm{b}}$ \\ a Dipartimento di Scienze Matematiche, Politecnico di Torino, corso Duca degli Abruzzi 24, 10129 Torino, Italy \\ b Dipartimento di Matematica "Francesco Brioschi", Politecnico di Milano, via Bonardi 9, 20133 Milano, Italy
}

\section{ABSTRACT}

Let $k$ be an algebraically closed field of characteristic 0 and let $\mathcal{H} i l b G\left(\mathbb{P}{ }_{k}^{N}\right)$ be the open locus of the Hilbert scheme $\mathcal{H} i l b d(\mathbb{P} k)$ corresponding to Gorenstein subschemes. We proved in several previous papers that $\mathcal{H i l b} G\left(\mathbb{P}{ }_{k}\right)$ is irreducible for $d \leqslant 10$ and $N \geqslant$ 1 , characterizing its singular locus. In the present paper we prove that also $\mathcal{H i l b} G_{1}\left(\mathbb{P}_{k}^{N}\right)$ is irreducible for each $N \geqslant 1$. We also give some results about its singular locus.

\section{Introduction and notation}

Let $k$ be an algebraically closed field of characteristic 0 and let $\mathcal{H} i b_{d}\left(\mathbb{P}_{k}^{N}\right)$ be the Hilbert scheme of 0-dimensional subschemes of $\mathbb{P}_{k}^{N}$ of degree $d$. A scheme $X \in \mathcal{H} i b_{d}\left(\mathbb{P}_{k}^{N}\right)$ is said to be smoothable if it is in the closure $\mathcal{H} i b_{d}^{\text {gen }}\left(\mathbb{P}_{k}^{N}\right)$ inside $\mathcal{H} i l b_{d}\left(\mathbb{P}_{k}^{N}\right)$ of the locus of reduced schemes $\mathcal{R}$. Notice that $\mathcal{R}$ is birational to a suitable open subset of the $d$ th symmetric product of $\mathbb{P}_{k}^{N}$, thus it is irreducible of dimension $d N$. In particular the irreducibility of $\mathcal{H} i b_{d}\left(\mathbb{P}_{k}^{N}\right)$ is equivalent to the smoothability of all 0 -dimensional subschemes of $\mathbb{P}_{k}^{N}$ of degree $d$.

It is known that $\mathcal{H i l b} b_{d}\left(\mathbb{P}_{k}^{N}\right)$ is actually irreducible if $N \leqslant 2$ (see [16] where a more general result is proven). On the other hand the reducibility of $\mathcal{H i l b} b_{d}\left(\mathbb{P}_{k}^{N}\right)$ for $d$ large enough with respect to $N$ is proved in [19]. The first explicit example of reducible punctual Hilbert scheme is $\mathcal{H i l b} b_{8}\left(\mathbb{P}_{k}^{4}\right)$ (see [15]). More recently, in [4], the authors showed that $\mathcal{H} i l b_{8}\left(\mathbb{P}_{k}^{N}\right), N \geqslant 4$, has exactly two irreducible components.

We now focus our attention on a smaller locus inside $\mathcal{H i l b}\left(\mathbb{P}_{k}^{N}\right)$, i.e. the set $\mathcal{H i l b}_{d}^{G}\left(\mathbb{P}_{k}^{N}\right)$ of points representing schemes which are Gorenstein. Such a locus is actually open inside $\mathcal{H i l b}_{d}\left(\mathbb{P}_{k}^{N}\right)$ (see [28]) and

\author{
Article history: \\ Received 6 July 2013 \\ Received in revised form 31 \\ December 2013 \\ Available online 17 January 2014 \\ Communicated by A.V. Geramita
}

\footnotetext{
The authors are members of GNSAGA group of INdAM. They are supported by the framework of PRIN 2010-2011 "Geometria sulle varietà algebriche", cofinanced by MIUR.

* Corresponding author.

E-mail addresses: gianfranco.casnati@polito.it (G. Casnati), roberto.notari@polimi.it (R. Notari).
} 
$\mathcal{R} \subseteq \mathcal{H i l b} b_{d}^{G}\left(\mathbb{P}_{k}^{N}\right)$, thus $\mathcal{H} i l b_{d}^{G, g e n}\left(\mathbb{P}_{k}^{N}\right):=\mathcal{H} i l b_{d}^{G}\left(\mathbb{P}_{k}^{N}\right) \cap \mathcal{H i l b} b_{d}^{g e n}\left(\mathbb{P}_{k}^{N}\right)$ is irreducible of dimension $d N$ and open in $\mathcal{H i l b}_{d}^{G}\left(\mathbb{P}_{k}^{N}\right)$.

On the one hand in [21], Lemma 6.21 , the authors claimed the reducibility of $\mathcal{H i l b} b_{4}^{G}\left(\mathbb{P}_{k}^{6}\right)$ (see also [9]) and suggested that $d=14$ could be the lower degree for which $\mathcal{H} i b_{d}^{G}\left(\mathbb{P}_{k}^{N}\right)$ is reducible. On the other hand $\mathcal{H} i l b_{d}^{G}\left(\mathbb{P}_{k}^{N}\right.$ ) is irreducible when $d \leqslant 10$ (see [8] and [9]) and for $N \leqslant 3$ without restrictions on $d$ (see e.g. [9], Corollary 2.6).

In the present paper we make a further step confirming the irreducibility of $\mathcal{H} i l b_{d}^{G}\left(\mathbb{P}_{k}^{N}\right)$ for $d \leqslant 13$ by proving the following

Main Theorem. The locus $\mathcal{H} i b_{11}^{G}\left(\mathbb{P}_{k}^{N}\right)$ is irreducible for $d \leqslant 11$.

The above result has an interesting consequence regarding secant varieties of Veronese embeddings. More precisely in [17] the author conjectures that the ideal of the 2nd secant variety (the variety of secant lines) of the $d$ th Veronese embedding of $\mathbb{P}_{k}^{n}$ is generated by the $3 \times 3$ minors of the $i$ th catalecticant matrix for $2 \leqslant i \leqslant d-2$. Such a conjecture was confirmed in [27]. As pointed out in [3], Section 8.1, the above Main Theorem allows to extend the above result as follows: if $r \leqslant 11,2 r \leqslant d$ and $i=r, \ldots, d-r$, then the set-theoretic equations of the $r$ th secant variety of the $d$ th Veronese embedding of $\mathbb{P}_{k}^{n}$ are given by the $(r+1) \times(r+1)$ minors of the $i$ th catalecticant matrix.

Notice that each $X \in \mathcal{H} i b_{d}^{G}\left(\mathbb{P}_{k}^{N}\right)$ is affine, thus it is isomorphic to $\operatorname{spec}(A)$ for a suitable Artinian, Gorenstein $k$-algebra (throughout this paper, a $k$-algebra is an associative, commutative and unitary algebra over $k$ ). In particular a first necessary step is to deal with such kind of algebras. Indeed, as pointed out in Lemma 2.2 of [8], the smoothability of the scheme $X:=\operatorname{spec}(A)$ does not depend on its embedding, but only on its intrinsic structure, i.e. only on the underlying algebra $A$. In particular, throughout the whole paper we will say that a $k$-algebra $A$ is smoothable if the same is true for the corresponding scheme $X:=\operatorname{spec}(A)$ with respect to a suitable projective embedding (and then, with respect to all ones).

The description of Artinian $k$-algebras is classical in commutative algebra and related fields. Since each Artinian $k$-algebra is a direct sum of local ones one can restrict its attention to local, Artinian $k$-algebras. A first invariant of any local Artinian $k$-algebra $A$ is its $\operatorname{dimension} d:=\operatorname{dim}_{k}(A)$ as $k$-vector space. Some general classification results are known for low values of $d$, namely $d \leqslant 6$ (e.g., see [23-25]). As $d$ increases the picture is not easy to handle with the same methods (see [4,26] and the references therein), unless we introduce some extra technical hypothesis. E.g., if $A$ is assumed to be Gorenstein, then a complete classification is available up to $d \leqslant 9$ (see [5]).

A second invariant which turns out to be extremely useful for such a classification is the Hilbert function $H_{A}$ of $A$, i.e. the Hilbert function of the associated graded ring

$$
\operatorname{gr}(A):=\bigoplus_{i=0}^{s} \mathfrak{M}^{i} / \mathfrak{M}^{i+1},
$$

where $\mathfrak{M}$ is the maximal ideal of $A$ and $s$ is the greatest integer $h$ such that $\mathfrak{M}^{h} \neq 0$.

Also with the Gorenstein hypothesis we are completely unable to achieve a classification up to isomorphisms as $d$ increases. For example, when $d \geqslant 10$ is not possible to classify in the above sense algebras with Hilbert function $(1, n, 4,1), n \geqslant 4$ : indeed Macaulay's theory of inverse systems and the results proven in [12] show that such a classification is actually strictly related to the classification up to projectivities of cubic surfaces in $\mathbb{P}_{k}^{3}$.

Anyhow, though it is not possible to classify all algebras for $d \geqslant 10$, the aforementioned relation actually represents a helpful structure result. For this reason, in order to better understand what follows, we spend here very few words on the aforementioned Macaulay's correspondence, postponing a more comprehensive résumé of such a theory to Section 2. Each local, Artinian, Gorenstein $k$-algebra $A$ can be obviously represented as a quotient of the form $k\left[\left[x_{1}, \ldots, x_{n}\right]\right] / J$ for a suitable ideal $J \subseteq\left(x_{1}, \ldots, x_{n}\right)^{2}$. Macaulay showed 
that if we look at $k\left[\left[x_{1}, \ldots, x_{n}\right]\right]$ as acting on $k\left[y_{1}, \ldots, y_{n}\right]$ via derivation (i.e. we identify $x_{i}$ with $\partial / \partial y_{i}, i$ $=1, \ldots, n)$, then each such $J$ is the annihilator $\operatorname{Ann}(F)$ of a suitable apolar polynomial $F \in k\left[y_{1}, \ldots, y_{n}\right]$ whose degree $s$ is exactly the maximum integer $s$ such that $\mathfrak{M}^{s} \neq 0$, the so-called socle degree $\operatorname{sdeg}(A)$ of $A$.

In this setup, the main result of [12] is that $A$ satisfies $H_{A}=(1, n, m, 1)$ if and only if $A k\left[\left[x_{1}, \ldots, x_{n}\right]\right] / \cong$ $\operatorname{Ann}\left(F_{3}+{ }_{i=m+1}^{n} y_{i}^{2}\right)$, fora suitable cubic form $F_{3} \in k\left[y_{1}, \ldots, y_{m}\right]$. Beside the interest of the above result in itself, it has several applications. E.g., in [6], we checked that such a decomposition of the apolar polynomial $F$ yields the smoothability of $A$.

Such a result throws some light on the question of the irreducibility of $\mathcal{H} i l b_{d}^{G}\left(\mathbb{P}_{k}^{N}\right)$. Along these lines the papers $[7,10]$ and the results proved in Section 3 generalizing the aforementioned decomposition of the apolar polynomial allow us to prove the smoothability of all the $k$-algebras $A$ with $\operatorname{dim}_{k}(A)=11$ proving the Main Theorem above.

The results on the decomposition of the apolar polynomial allow us to give an almost complete descrip-tion of the singular locus of $\mathcal{H} i b b_{11}^{G}\left(\mathbb{P}_{k}^{N}\right)$ in Section 6. Indeed, as for the smoothability, the property of a scheme $X=$ $\operatorname{spec}(A) \subseteq \mathbb{P}_{k}^{N}$ of representing a singular point of $\mathcal{H} i l b_{d}^{G}\left(\mathbb{P}_{k}^{N}\right)$ can be checked on its irreducible components and it depends only on $A$ and not on the embedding of $X$ (see [9]). Making use also of the results proven in [8] and [9], we can focus our attention on algebras $A$ with Hilbert function either $(1,4,3,2,1)$ or $(1,4,2,2,1)$ or $(1,5$, $3,1,1)$ or $(1,5,4,1)$ or $(1,6,3,1)$ or $(1,4,4,1,1)$. For all but the last case we are able to prove whether $X=$ $\operatorname{spec}(A)$ represents a singular point or not.

The analysis of the last case $(1,4,4,1,1)$ will be carried out as an example in the forthcoming paper [10] since it needs some extra results and a particular care.

\subsection{Notation}

A $k$-algebra is an associative, commutative and unitary algebra over $k$. For each $N \in \mathbb{N}$ we set $S[N]:=$ $k\left[\left[x_{1}, \ldots, x_{N}\right]\right]$ and $P[N]:=k\left[y_{1}, \ldots, y_{N}\right]$. We denote by $S[N]_{q}\left(\right.$ resp. $\left.P[N]_{q}\right)$ the homogeneous component of degree $q$ of such a graded $k$-algebra, and we set $S[N]_{\leqslant q}:={ }_{i=1}^{q} S\left[\bigoplus_{i}\left(\right.\right.$ resp. $\left.P[n]_{\leqslant q}:={ }_{i=1}^{q} P[n]_{i}\right)$. . $+:=\left(x_{1}, \ldots, x_{n}\right) \subseteq S[n]$. The ideal $S[N]_{+}$is the unique maximal ideal $\left(x_{1}, \ldots, x_{N}\right) \subseteq S[N]$.

A local ring $R$ is Gorenstein if its injective dimension as $R$-module is finite. An arbitrary ring $R$ is called Gorenstein if $R_{\mathfrak{M}}$ is Gorenstein for every maximal ideal $\mathfrak{M} \subseteq R$. A scheme $X$ is Gorenstein if and only if for each point $x \in X$ the $\operatorname{ring} \mathcal{O}_{X, x}$ is Gorenstein.

For each numerical polynomial $p(t) \in \mathbb{Q}[t]$, we denote by $\mathcal{H} i b_{p(t)}\left(\mathbb{P}_{k}^{N}\right)$ the Hilbert scheme of closed subschemes of $\mathbb{P}_{k}^{N}$ with Hilbert polynomial $p(t)$. With abuse of notation we will denote by the same symbol both a closed point in $\mathcal{H} i l b_{p(t)}\left(\mathbb{P}_{k}^{N}\right)$ and the corresponding subscheme of $\mathbb{P}_{k}^{N}$. We denote by $\mathcal{H} i l b_{p(t)}^{G}\left(\mathbb{P}_{k}^{N}\right)$ the locus of points representing Gorenstein schemes.

If $\gamma:=\left(\gamma_{1}, \ldots, \gamma_{N}\right) \in \mathbb{N}^{N}$ is a multi-index, then we set $t^{\gamma}:=t_{1}^{\gamma_{1}} \cdots t_{N}^{\gamma_{N}} \in k\left[t_{1}, \ldots, t_{N}\right]$.

For all the other notations and results we refer to [18].

\section{Some facts on Macaulay's correspondence}

Let $A$ be a local, Artinian $k$-algebra with maximal ideal $\mathfrak{M}$. The socle degree $\operatorname{sdeg}(A)$ of $A$ is the greatest integer $s$ such that $\mathfrak{M}^{s} \neq 0$. The Hilbert function $H_{A}$ of $A$ is the Hilbert function of $\operatorname{gr}(A)$. We know that $A \cong S[n] / J$ for a suitable ideal $J \subseteq S[n]_{+}^{2} \subseteq S[n]$, where $n:=\operatorname{dim}_{k}\left(\mathfrak{M} / \mathfrak{M}^{2}\right)=H_{A}(1)$.

Each element $f \in S[n]$ can be decomposed as $f=\sum_{i=0}^{\infty} f_{i}$ where $f_{i} \in S[n]_{i}$. The smallest integer $i$ such that $f_{i} \neq 0$ is called the order of $f$ and it is denoted by ord $(f)$. Moreover $f_{\text {ord }(f)}$ is called the lower degree form of $f$. It will be denoted in what follows with $\operatorname{ldf}(f)$. 
If $f \in J$, then $\operatorname{ord}(f) \geqslant 2$. The lower degree form ideal $\operatorname{ldf}(J)$ associated to $J$ is

$$
\operatorname{ldf}(J):=(\operatorname{ldf}(f) \mid f \in J) \subseteq S[n] .
$$

It is well known that $\operatorname{gr}(A) \cong S[n] / \operatorname{ldf}(J)$.

We have an action of $S[n]$ over $P[n]$ given by partial derivation defined by identifying $x_{i}$ with $\partial / \partial y_{i}$. Hence

$$
x^{\alpha} \circ y^{\beta}:= \begin{cases}\alpha !\left(\begin{array}{l}
\beta \\
\alpha
\end{array}\right) y^{\beta-\alpha} & \text { if } \beta \geqslant \alpha, \\
0 & \text { if } \beta \ngtr \alpha .\end{cases}
$$

Such an action endows $P[n]$ with a structure of module over $S[n]$. If $J \subseteq S[n]$ is an ideal and $M \subseteq P[n]$ is a $S[n]$-submodule we set

$$
\begin{aligned}
J^{\perp} & :=\{F \in P[n] \mid g \circ F=0, \forall g \in J\}, \\
\operatorname{Ann}(M) & :=\{g \in S[n] \mid g \circ F=0, \forall F \in M\} .
\end{aligned}
$$

For the following results see e.g. [14,20] and the references therein. Macaulay's theory of inverse system is based on the fact that such constructions $J \mapsto J^{\perp}$ and $M \mapsto \operatorname{Ann}(M)$ give rise to a reversing inclusion bijection between ideals $J \subseteq S[n]$ such that $S[n] / J$ is a local, Artinian $k$-algebra and finitely generated $S[n]$-submodules $M \subseteq P[n]$. In this bijection Gorenstein algebras $A$ with $\operatorname{sdeg}(A)=s$ correspond to cyclic $S[n]$-submodules $\langle F$ \rangle$_{S[n]} \subseteq P[n]$ generated by a polynomial $F$ of degree $s$. We simply write $\operatorname{Ann}(F)$ instead of $\operatorname{Ann}\left(\langle F\rangle_{S[n]}\right)$.

On the one hand, given a $S[n]$-module $M$, we define, for $q \in \mathbb{N}$,

$$
\operatorname{tdf}(M)_{q}:=\frac{M \cap P[n]_{\leqslant q}+P[n]_{\leqslant q-1}}{P[n]_{\leqslant q-1}}
$$

and we set $\operatorname{tdf}(M):=\bigoplus_{q=0}^{\infty} \operatorname{tdf}(M)_{q} \cdot \operatorname{tdf}(M)$ can be interpreted as the $S[n]$-submodule of $P[n]$ generated by the top degree forms of all the polynomials in $M$.

We have $\operatorname{ldf}(\operatorname{Ann}(M))=\operatorname{Ann}(\operatorname{tdf}(M))($ see [14]: see also [12], Formulas (2) and (3)) whence

$$
\operatorname{gr}(S[n] / \operatorname{Ann}(M)) \cong S[n] / \operatorname{ldf}(\operatorname{Ann}(M)) \cong S[n] / \operatorname{Ann}(\operatorname{tdf}(M))
$$

Thus

$$
H_{S[n] / \operatorname{Ann}(M)}(q)=\operatorname{dim}_{k}\left(\operatorname{tdf}(M)_{q}\right)
$$

We say that $M$ is non-degenerate if $H_{S[n] / \operatorname{Ann}(M)}(1)=\operatorname{dim}_{k}\left(\operatorname{tdf}(M)_{1}\right)=n$, i.e. if and only if the classes of $y_{1}, \ldots, y_{n}$ are linearly independent in $\operatorname{tdf}(M)$.

Let $A$ be Gorenstein with $s:=\operatorname{sdeg}(A)$, so that $\operatorname{Soc}(A)=\mathfrak{M}^{s} \cong k$. In particular $A \cong S[n] / \operatorname{Ann}(F)$, where $F:=\sum_{i=0}^{s} F_{i}, F_{i} \in P[n]_{i}$ and, trivially, we can always assume that $F_{0}=0$. It is easy to check that, for given $J=\operatorname{Ann}(F)$, it also holds $J=\operatorname{Ann}(F+\sigma \circ F)$ for every $\sigma \in S[n]$. Hence, it makes sense to look for an easier polynomial $G$ such that $\operatorname{Ann}(G)=\operatorname{Ann}(F)$.

Lemma 2.1. Let $s \geqslant 2, F, \widehat{F} \in P[n]_{\leqslant s}$ be such that $F-\widehat{F} \in P[n]_{\leqslant 1}$. If $\operatorname{Ann}(F) \subseteq S[n]_{+}^{2}$, then $\operatorname{Ann}(F)=$ $\operatorname{Ann}(\widehat{F})$. 
Proof. From $\operatorname{Ann}(F) \subseteq S[n]_{+}^{2}$, it follows $\operatorname{Ann}(F) \subseteq \operatorname{Ann}(\widehat{F})$. In fact, $\sigma \circ H=0$ for each $H \in P[n]_{\leqslant 1}$ and every $\sigma$ of order at least 2 . The same argument shows that each $\sigma \in \operatorname{Ann}(\widehat{F})$ of order $\geqslant 2$ belongs to $\operatorname{Ann}(F)$.

Assume by contradiction that $\sigma \in \operatorname{Ann}(\widehat{F}) \backslash \operatorname{Ann}(F)$. Then, $\sigma$ has order 1 , and $\sigma \circ F=\sigma \circ F_{1}=\lambda \neq 0$. By degree reasons, $\lambda \in k$. Let $\tau \in S[n]$ be of order $s$ (thus at least 2) such that $\tau \circ F_{s}=-\lambda$. It follows that $\sigma+\tau \in \operatorname{Ann}(F)$ has order 1 , and so we get a contradiction because $\operatorname{Ann}(F) \subseteq S[n]_{+}^{2}$. Hence $\operatorname{Ann}(F)=$ $\operatorname{Ann}(\widehat{F})$ as claimed.

Summarizing the previous lemma, if $J=\operatorname{Ann}(F) \subseteq S[n]_{+}^{2}$, then there exists $G=\sum_{i=2}^{s} G_{i}$ such that $J=\operatorname{Ann}(G)$. We will always make such an assumption in what follows.

We have a filtration with proper ideals (see [20])

$$
C(0):=\operatorname{gr}(A) \supset C(1) \supseteq C(2) \supseteq \cdots \supseteq C(s-2) \supseteq C(s-1):=0 .
$$

The quotients $Q(a):=C(a) / C(a+1)$ are reflexive graded $\operatorname{gr}(A)$-modules. Their Hilbert function $H_{Q(a)}$ of $Q(a)$ is symmetric around $(s-a) / 2$ (see [20]). In general $\operatorname{gr}(A)$ is no more Gorenstein, but the first quotient

$$
G(A):=Q_{A}(0) \cong S[n] / \operatorname{Ann}\left(F_{s}\right)
$$

is characterized by the property of being the unique (up to isomorphism) graded Gorenstein quotient $k$-algebra of $\operatorname{gr}(A)$ with the same socle degree.

The Hilbert function of $A$ satisfies

$$
H_{A}(i)=H_{\operatorname{gr}(A)}(i)=\sum_{a=0}^{s-2} H_{Q_{A}(a)}(i), \quad i \geqslant 0 .
$$

Since $H_{A}(0)=H_{G(A)}(0)=1$, it follows that if $a \geqslant 1$, then $Q_{A}(a)_{0}=0$, whence $Q_{A}(a)_{i}=0$ when $i \geqslant s-a$ for the same values of $a$. In particular

$$
H_{\operatorname{gr}(A) / C_{A}(a+1)}(i)=H_{S[n] / \widehat{C}_{A}(a+1)}(i)=\sum_{\alpha=0}^{a} H_{Q_{A}(\alpha)}(i), \quad i \geqslant 0 .
$$

\section{Smoothability and apolar polynomial}

In this section we recall and prove some results related to the apolar polynomial of a local, Artinian, Gorenstein $k$-algebra which will be helpful later on both from the classification and the smoothability viewpoint.

We start by recalling that it is always possible (see [20], Theorems 5.3A and 5.3B) to choose a system of generators of $S[n]_{+}$such that $F$ satisfies $F_{i} \in P\left[f_{i}\right]$, where

$$
f_{i}:=\sum_{a=0}^{s-i} H_{Q(a)}(1)
$$

(so that $n=H_{A}(1)=f_{2}$ ). More precisely one can also obtain the following result.

Proposition 3.1. Let $A$ be a local, Artinian, Gorenstein $k$-algebra. If $n:=H_{A}(1)$ and $s:=\operatorname{sdeg}(A)$, then

$$
A \cong S[n] / \operatorname{Ann}(F)
$$

where $F:=\sum_{i=2}^{s} F_{i}+\sum_{j=f_{3}+1}^{n} y_{j}^{2}, F_{i} \in P\left[f_{i}\right]_{i}, i \geqslant 3$, and $F_{2} \in P\left[f_{3}\right]_{2}$. 
Proof. See [10], Proposition 3.2.

The above proposition allows us to reduce the study of algebras associated to polynomials of the form $F=G+x_{n}^{2} \in P[n]$ with $s>2$ and $G \in P[n-1]$ to $S[n-1] / \operatorname{Ann}(G)$. Indeed Corollary 3.2 of [7] yields

$$
\operatorname{Ann}(F)=\operatorname{Ann}(G) S[n]+\left(x_{n}^{2}-2 \sigma, x_{i} x_{n}\right)_{1 \leqslant i \leqslant n-1}
$$

where $\sigma \in S[n-1]$ has order $s$ and $\sigma \circ G=1$. Moreover

$$
H_{S[n] / \operatorname{Ann}(F)}(q)= \begin{cases}H_{S[n-1] / \operatorname{Ann}(G)}(q) & \text { if } q \geqslant 2 \\ H_{S[n-1] / \operatorname{Ann}(G)}(q)+1 & \text { if } q=1\end{cases}
$$

In fact, if $q>2$, Formula (2) gives

$$
H_{S[n] / \operatorname{Ann}(F)}(q)=\operatorname{dim}_{k}\left(\operatorname{tdf}(F)_{q}\right)=\operatorname{dim}_{k}\left(\operatorname{tdf}(G)_{q}\right)=H_{S[n-1] / \operatorname{Ann}(G)}(q) .
$$

The same equality still holds for $q=2$, because $\operatorname{tdf}(G)_{2}=\operatorname{tdf}\left(G+y_{n}^{2}\right)_{2}$. Finally, if $q=1$ we know that $y_{n} \in \operatorname{tdf}(F)_{q} \backslash \operatorname{tdf}(G)_{q}$ and $\operatorname{tdf}(F)_{q}=\operatorname{tdf}(G)_{q}+\left\langle y_{n}\right\rangle$.

Let $F$ and $G$ be as above. We want to relate the smoothability of the two schemes $X:=\operatorname{spec}(S[n] /$ $\operatorname{Ann}(F))$ and

$$
\widehat{X}:=\operatorname{spec}\left(\frac{S[n-1]}{\operatorname{Ann}(G)}\right) \cong \operatorname{spec}\left(\frac{S[n]}{\operatorname{Ann}(G)+\left(x_{n}\right)}\right) .
$$

To this purpose we first embed such schemes in $\mathbb{A}_{k}^{n}$ via the natural monomorphism $k\left[x_{1}, \ldots, x_{n}\right] \subseteq S[n]$. Both the schemes are then supported on the origin. Let us now consider the scheme $\mathcal{X} \subseteq \mathbb{A}_{k}^{1} \times \mathbb{A}_{k}^{n}$ defined by the ideal

$$
\mathcal{J}:=\operatorname{Ann}(G) k[b] \otimes S[n]+\left(x_{n}^{2}-b x_{n}-2 \sigma, x_{i} x_{n}\right)_{1 \leqslant i \leqslant n-1}
$$

inside $k[b] \otimes S[n]$. The map $\chi: \mathcal{X} \rightarrow \mathbb{A}_{k}^{1}$ induced by the projection on the first factor is thus a family of closed subschemes of $\mathbb{P}_{k}^{n}$.

If $\left(b, a_{1}, \ldots, a_{n}\right)$ is in the support of $\mathcal{J}$, then $\left(a_{1}, \ldots, a_{n-1}, 0\right) \in \widehat{X}$. Thus $a_{1}=\cdots=a_{n-1}=0$ and $a_{n}^{2}=$ $b a_{n}$ since $\sigma(0, \ldots, 0)=0$. It follows that the reduced structure of $\mathcal{X}$ is given by the ideal $\left(x_{1}, \ldots, x_{n-1}, x_{n}-b\right) \cap$ $\left(x_{1}, \ldots, x_{n}\right)$, whence $\mathcal{X}$ coincides with its closure $\mathcal{X} \subseteq \mathbb{A}_{k}^{1} \times \mathbb{P}_{k}^{n}$. We claim that $\chi$ is flat: thanks to the above discussion and to [18] Theorem, III.9.9, it suffices to show that its fibres have constant degree in $\mathbb{P}_{k}^{n}$.

Thanks to Equalities (5) and (6) the special fibre of $\mathcal{X}$ over 0 is exactly $X$ and its degree is $d:=\operatorname{deg}(X)=$ $\operatorname{deg}(\widehat{X})+1$.

Lemma 3.2. If $b \neq 0$, then $\mathcal{J}_{b}=\left(x_{1}, \ldots, x_{n-1}, x_{n}-b\right) \cap \mathcal{J}_{b}^{\prime} \subseteq S[n]$, where

$$
\mathcal{J}_{b}^{\prime}:=\operatorname{Ann}(G) S[n]+\left(x_{i} x_{n}, b x_{n}+2 \sigma\right)_{1 \leqslant i \leqslant n} .
$$

Proof. Set $\mathcal{J}_{b}^{\prime \prime}:=\left(x_{1}, \ldots, x_{n-1}, x_{n}-b\right)$. Trivially $\mathcal{J}_{b} \subseteq \mathcal{J}_{b}^{\prime \prime}$ and $\mathcal{J}_{b}^{\prime}=\mathcal{J}_{b}+\left(x_{n}^{2}\right)$, thus the modular law (see [1]) implies $\mathcal{J}_{b}^{\prime \prime} \cap \mathcal{J}_{b}^{\prime}=\mathcal{J}_{b}^{\prime \prime} \cap\left(\mathcal{J}_{b}+\left(x_{n}^{2}\right)\right)=\mathcal{J}_{b}+\mathcal{J}_{b}^{\prime \prime} \cap\left(x_{n}^{2}\right)$. We have $\mathcal{J}_{b}^{\prime \prime} \cap\left(x_{n}^{2}\right)=\mathcal{J}_{b}^{\prime \prime}\left(x_{n}^{2}\right)=$ $\left(x_{1} x_{n}^{2}, \ldots, x_{n-1} x_{n}^{2},\left(x_{n}-b\right) x_{n}^{2}\right)$. By the definition of $\mathcal{J}_{b}$ we have $x_{i} x_{n}^{2} \in \mathcal{J}_{b}, i=1, \ldots, n-1$, and $x_{n} \sigma \in \mathcal{J}_{b}$. It follows that $\left(x_{n}-b\right) x_{n}^{2}=\left(x_{n}^{2}-b x_{n}\right) x_{n} \in\left(x_{n} \sigma\right)+\mathcal{J}_{b}=\mathcal{J}_{b}$, thus $\mathcal{J}_{b}^{\prime \prime} \cap\left(x_{n}^{2}\right) \subseteq \mathcal{J}_{b}$. In particular, $\mathcal{J}_{b}+\mathcal{J}_{b}^{\prime \prime} \cap\left(x_{n}^{2}\right)=\mathcal{J}_{b}$, hence the statement is proved. 
Now we come back to the family $\mathcal{X} \rightarrow \mathbb{A}_{k}^{1}$. The ideals $\mathcal{J}_{b}^{\prime}$ and $\mathcal{J}_{b}^{\prime \prime}$ are coprime for $b \neq 0$ : indeed $u:=-x_{n}^{2}+2 b x_{n}-4 \sigma \in \mathcal{J}_{b}^{\prime}, v:=4 \sigma+\left(x_{n}-b\right)^{2} \in \mathcal{J}_{b}^{\prime \prime}$ and $u+v=b^{2}$. Hence Lemma 3.2 implies that the fibre $\mathcal{X}_{b}$ over $b \neq 0$ splits as $\operatorname{spec}\left(S[n] / \mathcal{J}_{b}^{\prime}\right) \cup \operatorname{spec}(k)$. Moreover $x_{i} \sigma \in S[n-1]$ for each $i=1, \ldots, n-1$ and it has order $s+1$, thus it is contained in $\operatorname{Ann}(G)$. We conclude that

$$
S[n] / \mathcal{J}_{b}^{\prime} \cong S[n] / \operatorname{Ann}(G) S[n]+\left(x_{i} x_{n}, b x_{n}+2 \sigma\right)_{1 \leqslant i \leqslant n} \cong S[n-1] / \operatorname{Ann}(G) .
$$

Taking into account Eq. (5), we deduce that, for each $b \neq 0$, we have

$$
\operatorname{dim}_{k}\left(S[n] / \mathcal{J}_{b}^{\prime}\right)=\operatorname{dim}_{k}(S[n-1] / \operatorname{Ann}(G))=\operatorname{dim}_{k}(S[n] / \operatorname{Ann}(F))-1
$$

It follows that the degree of the fibre $\mathcal{X}_{b}$ is constantly $d$ also for $b \neq 0$, whence $\chi$ is a flat family of closed subschemes in $\mathbb{P}_{k}^{n}$.

As pointed out in the Introduction, recall that a local, Artinian, Gorenstein $k$-algebra $A$ with $\operatorname{dim}_{k}(A)=d$ is smoothable if and only if $X \cong \operatorname{spec}(A) \in \mathcal{H} i l b_{d}^{G, g e n}\left(\mathbb{P}_{k}^{N}\right)$ and such a notion does not depend on the embedding of $X$, but only on the intrinsic structure of $X$, i.e. on $A$.

Theorem 3.3. Let $F=G+y_{n}^{2}$. Let us denote by $\operatorname{Ann}(G)$ the annihilator of $G$ inside $S[n-1]$. If $S[n-1] /$ $\operatorname{Ann}(G)$ is smoothable, then the same holds for $S[n] / \operatorname{Ann}(F)$.

Proof. Consider the family $\chi: \mathcal{X} \rightarrow \mathbb{A}_{k}^{1}$ defined above. The universal property of the Hilbert scheme guarantees the existence of a map $i: \mathbb{A}_{k}^{1} \rightarrow \mathcal{H} i l b_{d}^{G}\left(\mathbb{P}_{k}^{n}\right)$ such that $i(0)=X$ and $i(b) \in \mathcal{H} i l b_{d}^{G, g e n}\left(\mathbb{P}_{k}^{n}\right)$ for $b \neq 0$, due to the hypothesis. Since this last scheme is closed inside $\mathcal{H i l b} b_{d}^{G}\left(\mathbb{P}_{k}^{n}\right)$ by definition, it finally follows that $X \in \mathcal{H i l b}{ }_{d}^{G, g e n}\left(\mathbb{P}_{k}^{n}\right)$.

As an immediate consequence of the above theorem and of Proposition 3.1 we finally obtain the following corollary.

Corollary 3.4. Let $A$ be a local, Artinian, Gorenstein k-algebra with $f_{3} \leqslant 3$. Then $A$ is smoothable.

Proof. Thanks to Proposition 3.1 we know $A \quad S[n] / \operatorname{Ann}\left(G+\sum_{j=f_{3}+1}^{n} y_{j}^{2}\right)$. Since $f_{3} \leqslant 3$, it follows that $S\left[f_{3}\right] / \operatorname{Ann}(G)$ is smoothable (e.g. see [8], Proposition 2.5), hence an iterated application of Theorem 3.3 yields that $A$ is smoothable too.

\section{The Gorenstein locus of the punctual Hilbert scheme}

We now prove the Main Theorem stated in the Introduction.

Theorem 4.1. The locus $\mathcal{H} i b_{d}^{G}\left(\mathbb{P}_{k}^{N}\right)$ is irreducible if $d \leqslant 11$.

Proof. It suffices to check that each irreducible scheme $X \cong \operatorname{spec}(A) \in \mathcal{H} i b_{11}^{G}\left(\mathbb{P}_{k}^{N}\right)$ is smoothable. Thanks to Corollary 3.4 it suffices to check this fact for algebras $A$ with $f_{3} \geqslant 4$.

Recall that $H_{Q(a)}$ is symmetric around $(s-a) / 2$ where $s:=\operatorname{sdeg}(A)$. From the symmetry of $H_{Q(a)}$ we obtain

$$
\operatorname{dim}_{k}(G(A))+2 \sum_{a=1}^{s-3} H_{Q(a)}(1) \leqslant \operatorname{dim}_{k}(A) \leqslant 11
$$

where $G(A)=Q(0)$ as defined in Eq. (3). 
If $s \geqslant 5$, then $\operatorname{dim}_{k}(G(A)) \geqslant 4+2 H_{G(A)}(1)$, thus $2 f_{3}=2 \sum_{a=0}^{s-3} H_{Q(a)}(1) \leqslant 7$, whence $f_{3} \leqslant 3$. Thus we must restrict our attention on the cases $s=3$, In the first case, then $H_{Q(1)}$ is symmetric around 1 , hence

$H_{Q(1)}(2)=H_{Q(1)}(0)=0$. It follows that $f_{3}=H_{G(A)}(1)=H_{G(A)}(2)=H_{A}(2)$. In particular, if $f_{3} \geqslant 4$, then $H_{A}=(1,5,4,1)$. The smoothability of such algebras was proven in [6], Corollary 3.3.

Now assume $s=4$. Let $H_{G(A)}=\left(1, a_{1}, a_{2}, a_{1}, 1\right), H_{Q(1)}=\left(0, b_{1}, b_{1}, 0,0\right)$. If $a_{2}+b_{1} \geqslant 4$, and $H_{A}(1) \geqslant$ 4 , then $H_{A}=(1,4,4,1,1)$ necessarily. Now assume $a_{2}+b_{1} \leqslant 3$, whence $a_{2} \leqslant 3$. If $a_{2} \leqslant 2$, then $a_{1} \leqslant a_{2}$ thanks to Macaulay's growth theorem (see [2], theorem 4.2.10), hence $f_{3}=a_{1}+b_{1} \leqslant a_{2}+b_{1} \leqslant 3$.

Let $a_{2}=3$, so that $b_{1}=0$. Again Macaulay's growth theorem implies $a_{1} \leqslant 4$. Equality cannot hold, otherwise $\operatorname{dim}_{k}(G(A))=13$. Thus $f_{3}=a_{1} \leqslant 3$.

We now focus our attention on the schemes $X \subseteq \mathbb{P}_{k}^{N}$ such that $X \cong \operatorname{spec}(A)$ with $H_{A}=(1,4,4,1,1)$. We already pointed out that the smoothability of $X$ only depends on $A$. Thus it suffices to check that $X$ is smoothable with respect to a particular embedding, e.g. to the natural embedding $X \subseteq \mathbb{P}_{k}^{4}$ induced by $k\left[x_{1}, x_{2}, x_{3}, x_{4}\right] \longmapsto S[4] \rightarrow S[4] / J \cong A$.

Let $\mathcal{Z}_{4} \subseteq \mathcal{H} i l b_{11}^{G}\left(\mathbb{P}_{k}^{4}\right)$ be the locus of points representing irreducible schemes corresponding to algebras with Hilbert function $(1,4,4,1,1)$. We will show that $\mathcal{Z}_{4} \subseteq \mathcal{H i l b} b_{11}^{G, g e n}\left(\mathbb{P}_{k}^{4}\right)$. Recall that the tangent space to $\mathcal{H} i b_{11}^{G}\left(\mathbb{P}_{k}^{4}\right)$ at any point $Z$ is canonically identified with $H^{0}\left(Z, \mathcal{N}_{Z}\right), \mathcal{N}_{Z}$ being the normal sheaf of $Z \subseteq \mathbb{A}_{k}^{4}$.

Claim 4.2. $\mathcal{Z}_{4}$ is irreducible.

Claim 4.3. There exists a smoothable $X \in \mathcal{Z}_{4} \curvearrowright \mathcal{H} i l b_{11}^{G, g e n}\left(\mathbb{P}_{k}^{4}\right)$.

$$
\left.{ }_{X}\right)=44 \text {. }
$$

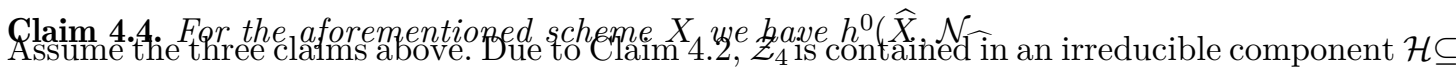
$\mathcal{H} i l b^{G}{ }_{11}\left(\mathbb{P}_{k}^{4}\right)$. If $\mathcal{H} \neq \mathcal{H} i l b_{11}{ }^{G, g e n}\left(\mathbb{P}^{4}{ }_{k}\right)$ each $X \in \mathcal{Z}_{4} \cap \mathcal{H} i l b_{11}{ }^{G, g e n}\left(\mathbb{P}_{k}^{4}\right)$ would be necessarily obstructed, whence $h^{0}\left(X, \mathcal{N}_{X}\right)>\operatorname{dim}\left(\mathcal{H} i b_{11}{ }^{G, g e n}\left(\mathbb{P}^{4}{ }_{k}\right)\right)=44$. In particular, such an inequality would hold true for the scherhe defined in Claim 4.3, contradicting Claim 4.4. Thus the theorem is completely proven.

We will now prove the claims above.

Proof of Claim 4.2. There exists a natural fibration $\mathcal{Z}_{4} \rightarrow \mathbb{P}_{k}^{4}$ associating to each scheme its supporting scheme. All the fibres of such a map are trivially isomorphic, thus $\mathcal{Z}_{4} \cong \mathbb{P}_{k}^{4} \times \mathcal{Z}_{4}(0), \mathcal{Z}_{4}(0)$ being the fibre over the point $[1,0,0,0,0] \in \mathbb{P}_{k}^{4}$. We claim that $\mathcal{Z}_{4}(0)$ is irreducible, whence we deduce the irreducibility of $\mathcal{Z}_{4}$.

Let $X:=\operatorname{spec}(A) \in \mathcal{Z}_{4}(0)$. Thus $A \cong S[4] / \operatorname{Ann}(F)$ where $\in P[4]$ has degree 4 . If $F_{4}$ is the top degree form of $F$, then $G(A) \cong S[4] / \operatorname{Ann}\left(F_{4}\right)$ (see Formula $(3)$ ). Since $H_{A}=(\mathbf{F}, 4,4,1,1)$, we deduce that $H_{G(A)}=(1,1$, $1,1,1)$, whence we deduce that $F_{4}=\ell^{4}$ for a suitable non-zero linear form. In particular, $F=\ell^{4}+G$ where $G$ $\in P[4]_{\leqslant 3}$.

Conversely let $V \subseteq P[4]$ be the scheme of polynomials of the form $\ell^{4}+G$ where $\ell$ is a linear form and $\operatorname{deg}(G) \leqslant 3 . V$ is trivially irreducible since it has naturally the structure of a vector bundle over the subspace $P$ $[4]_{\leqslant 3} \subseteq P[4]$. We assert that there exists a Zariski non-empty open subset $U \subseteq V$ such that $S[4] / \operatorname{Ann}\left(\ell^{4}+\right.$ $G)$ has Hilbert function $(1,4,4,1,1)$ if $\ell^{4}+G \in U$. Indeed $G(A) \cong S[4] / \operatorname{Ann}\left(\ell^{4}\right)$, thus $H_{G(A)}=(1,1,1,1,1)$. Decomposition (4) implies $H_{A}=(1, n, m, 1,1)$ with $1 \leqslant m \leqslant n \leqslant 4$.

Now let $\mathbb{A}_{k}^{70}$ be the affine space whose points represent polynomials of degree at most 4 in $k\left[x_{1}, x_{2}, x_{3}, x_{4}\right] \subseteq$ $S[4]$ and define

$$
W:=\left\{(F, g) \in V \times \mathbb{A}_{k}^{70} \mid g \in \operatorname{Ann}(F)\right\}
$$

We have $\left(\sum_{\alpha} a_{\alpha} y^{\alpha}, \sum_{\beta} b_{\beta} x^{\beta}\right) \in W$ if and only if 


$$
\sum_{\alpha, \beta} a_{\alpha} b_{\beta} x^{\beta} \circ y^{\alpha}=0
$$

thus $W$ is a closed subvariety of $V \times \mathbb{A}_{k}^{70}$. Moreover, we also have an induced projection $\pi: W \subseteq V \times \mathbb{A}_{k}^{70} \rightarrow V$ whose fibre over the point corresponding to an algebra with Hilbert function $(1, n, m, 1,1)$ is a linear subspace in $\mathbb{A}_{k}^{70}$ of dimension $67-m-n$. Set

$$
V_{h}:=\left\{F \in V \mid \operatorname{dim}\left(\pi^{-1}(F)\right) \geqslant 67-h\right\} \subseteq V .
$$

Due to [18], Exercise II.3.22, it follows that $V_{h}$ is closed. Since $1 \leqslant m \leqslant n \leqslant 4$ and, trivially, $V_{2} \subseteq V_{3} \subseteq$ $\cdots \subseteq V_{7} \subseteq V_{8}$ we also have $V_{8}=V$. Finally, it is easy to check that for each $m, n$ such that $1 \leqslant m \leqslant n \leqslant 4$

$$
y_{4}^{4}+\sum_{i=1}^{m-1} y_{i}^{3}+\sum_{i=m}^{3} y_{i}^{2} \in V_{m+n} \backslash V_{m+n-1}
$$

the first (resp. second) summation being empty if $m=1$ (resp. $m=4$ ). We conclude that $U:=V_{8} \backslash V_{7} \subseteq V$ is open and non-empty.

Let us consider the family of ideals

$$
\left\{(F, g) \in U \times \mathbb{A}_{k}^{70} \mid g \in \operatorname{Ann}(F)\right\}+\left(x_{1}, x_{2}, x_{3}, x_{4}\right)^{5} \subseteq U \times k\left[x_{1}, x_{2}, x_{3}, x_{4}\right]
$$

Arguing as in the construction of family $\chi$ in the previous Section 3, we obtain a family $\mathcal{X} \subseteq U \times \mathbb{A}_{k}^{4} \subseteq U \times \mathbb{P}_{k}^{4}$ which is flat over $U$. The universal property of the Hilbert scheme yields the existence of a natural map $U \rightarrow \mathcal{H i l b} b_{11}^{G}\left(\mathbb{P}_{k}^{4}\right)$ whose image is exactly $\mathcal{Z}_{4}(0)$. Since $U$ is irreducible the same is true for $\mathcal{Z}_{4}(0)$.

Proof of Claim 4.3. Let us consider in $k[b] \otimes S[4]$ the ideal

$$
\mathcal{J}:=\left(x_{3} x_{4}, x_{2} x_{4}, x_{1} x_{4}, x_{1}^{3}-x_{2}^{2}, x_{1} x_{2}+x_{3}^{2}, x_{1} x_{3}, x_{4}^{3}-b^{2} x_{4}+(b-1) x_{1}^{4}, x_{3}^{3}, x_{2}^{2} x_{3}, x_{2}^{3}\right)
$$

and consider the corresponding scheme $\mathcal{X} \subseteq \mathbb{A}_{k}^{1} \times \mathbb{A}_{k}^{4} \subseteq \mathbb{A}_{k}^{1} \times \mathbb{P}_{k}^{4}$, inducing a family $\chi: \mathcal{X} \rightarrow \mathbb{A}_{k}^{1}$.

The special fibre $\widehat{X}$ of $\mathcal{X}$ over 0 is $\operatorname{spec}(A)$ where $A=S[4] / J$ with

$$
J:=\left(x_{3} x_{4}, x_{2} x_{4}, x_{1} x_{4}, x_{1}^{3}-x_{2}^{2}, x_{1} x_{2}+x_{3}^{2}, x_{1} x_{3}, x_{4}^{3}-x_{1}^{4}, x_{3}^{3}, x_{2}^{2} x_{3}, x_{2}^{3}\right) .
$$

Let $a_{i}$ be the class of $x_{i}$ in $A$. We have $H_{A}=(1,4,4,1,1)$ with basis $1, a_{1}, a_{2}, a_{3}, a_{4}, a_{1}^{2}, a_{2} a_{3}, a_{3}^{2}, a_{4}^{2}$, $a_{1}^{3}, a_{1}^{4}$. In particular, $\operatorname{dim}_{k}(A)=11$. We also have

$a_{1} a_{3}=a_{1} a_{4}=a_{2} a_{4}=a_{3} a_{4}=0, \quad a_{1} a_{2}=-a_{3}^{2}, \quad a_{2}^{2}=a_{1}^{3}, \quad a_{1} a_{2}^{2}=a_{1}^{4}, \quad a_{2} a_{3}^{2}=a_{1}^{4}, \quad a_{4}^{3}=a_{1}^{4}$.

Let

$$
a=\alpha_{0}+\sum_{i=1}^{4} \alpha_{i} a_{i}+\alpha_{5} a_{1}^{2}+\alpha_{6} a_{2} a_{3}+\alpha_{7} a_{3}^{2}+\alpha_{8} a_{4}^{2}+\alpha_{9} a_{1}^{3}+\alpha_{10} a_{1}^{4}
$$

where $\alpha_{h} \in k, h=0, \ldots, 10$. Using the above relations it is easy to check that the condition $a \in \operatorname{Soc}(A)$ yields $\alpha_{h}=0, h=0, \ldots, 9$, thus $\operatorname{Soc}(A) \cong k$. We deduce that $A$ is Gorenstein. 
Now take $b \neq 0$ and define

$$
\begin{aligned}
\mathcal{J}^{\prime} & :=\left(x_{4}^{2}, x_{3} x_{4}, x_{2} x_{4}, x_{1} x_{4}, x_{1} x_{3}, x_{1} x_{2}+x_{3}^{2}, x_{1}^{3}-x_{2}^{2}, x_{3}^{3}, x_{2}^{2} x_{3}, x_{2}^{3},(b-1) x_{1}^{4}-b^{2} x_{4}\right), \\
\mathcal{J}^{\prime \prime} & :=\left(x_{1}, x_{2}, x_{3}, x_{4}^{2}-b^{2}\right) .
\end{aligned}
$$

One can check that $\mathcal{J}_{b}=\mathcal{J}_{b}^{\prime} \cap \mathcal{J}_{b}^{\prime \prime}$ if $b \neq 0$ (imitate the proof of Lemma 3.2). Thus, $\mathcal{X}_{b}$ with $b \neq 0$ is the disjoint union of the two simple points $[1,0,0,0, \pm b] \in \mathbb{P}_{k}^{4}$, corresponding to the ideal $\mathcal{J}_{b}^{\prime \prime}$, and of a smoothable scheme of degree $d-2$ supported on $[1,0,0,0,0] \in \mathbb{P}_{k}^{4}$ defined by $\mathcal{J}_{b}^{\prime}$. This last scheme is smoothable because

$$
S[4] / \mathcal{J}_{b}^{\prime} \cong S[3] /\left(x_{1} x_{3}, x_{1} x_{2}+x_{3}^{2}, x_{1}^{3}-x_{2}^{2}\right)=: A_{3,3,9}^{0}
$$

(see [8] for a proof of the smoothability of $A_{3,3,9}^{0}$ ). Thus, we can follow verbatim the same argument of the proof of Theorem 3.3. We conclude that $\widehat{X}$ is smoothable too.

Proof of Claim 4.4. Let $\widehat{X} \subseteq \mathbb{P}_{k}^{4}$ be the embedding induced by the identification $\mathbb{A}_{k}^{4}=\left\{x_{0} \neq 0\right\} \subseteq \mathbb{P}_{k}^{4}$. $\operatorname{dim}\left(\mathcal{H i l b} b_{11}^{G, g e n}\left(\mathbb{P}_{k}^{4}\right)\right)=44$ and we have to show that $h^{0}\left(\widehat{X}, \mathcal{N}_{\widehat{X}}\right)=44$. Recall that if $Z \cong \operatorname{spec}(S[N] / J) \subseteq \mathbb{A}_{k}^{N}$ is a Gorenstein punctual scheme, then

$$
h^{0}\left(Z, \mathcal{N}_{Z}\right)=\operatorname{dim}_{k}\left(S[N] / J^{2}\right)-\operatorname{dim}_{k}(S[N] / J)
$$

(see [8], Proposition 5.5).

In the case we are interested in, using any computer algebra software, it is immediate to obtain $h^{0}\left(\widehat{X}, \mathcal{N}_{\widehat{X}}\right)=44$, for the aforementioned scheme $X$, whence the assertion.

\section{Classification of algebras with Hilbert functions $(1, n, 2,2,1,1)$ and $(1, n, 3,2,1)$}

In this section we give a complete classification of local, Artinian, Gorenstein $k$-algebras $A$ such that $H_{A}$ is either $(1, n, 2,2,1,1)$ or $(1, n, 3,2,1)$. Besides its intrinsic interest, we will make partial use of such a classification in the next Section 6 , in order to deal with the singular locus of $\mathcal{H} i b_{11}^{G}\left(\mathbb{P}_{k}^{N}\right)$.

Assume first $H_{A}=(1, n, 2,2,1,1)$ where $n \geqslant 2$. Thus Decomposition (4), is

$$
(1,1,1,1,1,1)+(0,1,1,1,0,0)+(0, n-2,0,0,0,0) .
$$

Theorem 5.1. Let $n \geqslant 2$ be an integral number. If $A$ is a local, Artinian, Gorenstein, $k$-algebra with $H_{A}=$ $(1, n, 2,2,1,1)$, then $A \cong A_{n, 2,2, n+7}^{t}:=S[n] / J_{t}, t=1,2$, where

$$
\begin{aligned}
& J_{1}:=\left(x_{2}^{2}-x_{1}^{3}, x_{1}^{3} x_{2}, x_{i} x_{j}, x_{j}^{2}-x_{1}^{5}\right)_{i \geqslant 1, j \geqslant 3, i \neq j}, \\
& J_{2}:=\left(x_{2}^{2}-x_{1} x_{2}-x_{1}^{3}, x_{1}^{3} x_{2}, x_{i} x_{j}, x_{j}^{2}-x_{1}^{5}\right)_{i \geqslant 1, j \geqslant 3, i \neq j} .
\end{aligned}
$$

Moreover, $A_{n, 2,2, n+7}^{t} \cong A_{n^{\prime}, 2,2, n^{\prime}+7}^{t^{\prime}}$ if and only if $n=n^{\prime}$ and $t=t^{\prime}$.

Proof. Weknow that $A S[n]$ FAnn $(F)$ where $F=G+\sum_{j=2}^{n} y_{j}^{2}$ where $G \in P[2]$ (see Theorem 3.1). An iterated application of Equality (6) implies that both

$$
\operatorname{Ann}(F)=\operatorname{Ann}(G) S[n]+\left(x_{i} x_{j}, x_{j}^{2}-\sigma\right)_{i \geqslant 1, j \geqslant 3, i \neq j}
$$

where $\sigma \circ G=1$ and the Hilbert function of $S[2] / \operatorname{Ann}(G)$ is $(1,2,2,2,1,1)$. Thanks to [13], Theorem 2.8, up to automorphisms of $S[2], \operatorname{Ann}(G)$ can be put in one of the following forms 


$$
I_{1}:=\left(x_{2}^{2}-x_{1}^{3}, x_{1}^{3} x_{2}\right), \quad I_{1}^{\prime}:=\left(x_{2}^{2}-x_{1}^{2} x_{2} x_{1}^{3}, x_{1}^{3} x_{2}\right), \quad I_{2}:=\left(x_{2}^{2}-x_{1} x_{2}-x_{1}^{3}, x_{1}^{3} x_{2}\right) .
$$

Example 5.4 of [13] shows that $S[2] / I_{1} \cong S[2] / I_{2}^{\prime}$. It is easy to check that $I_{1}=\operatorname{Ann}\left(y_{1}^{5}+30 y_{1}^{2} y_{2}^{2}\right)$, $I_{2}=\operatorname{Ann}\left(y_{1}^{5}+30 y_{1}^{2} y_{2}^{2}+20 y_{1} y_{2}^{3}+5 y_{2}^{4}\right)$, thus we can choose $\sigma=x_{1}^{5} / 5$ !. The transformation $x_{j} \mapsto x_{j} / \sqrt{5}$ !, $j \geqslant 3$, finally yields the ideals $J_{1}$ and $J_{2}$ respectively.

It remains to show that $A_{n, 2,2, n+7}^{t} \cong A_{n^{\prime}, 2,2, n^{\prime}+7}^{t^{\prime}}$ if and only if $n=n^{\prime}$ and $t=t^{\prime}$. The "if" part is an easy check. So, we assume that $A_{n, 2,2, n+7}^{t} \cong A_{n^{\prime}, 2,2, n^{\prime}+7}^{t^{\prime}, 2,2, n^{\prime}+7}$.

Trivially $n=H_{A_{n, 2,2, n+7}^{t}}(1)=H_{A_{n^{\prime}, 2,2, n^{\prime}+7}^{t^{\prime}}}(1)=n^{\prime}$. It remains to prove that $A_{n, 2,2, n+7}^{1} \nsucceq A_{n, 2,2, n+7}^{2}$. Consider the set

$$
U_{t}:=\left\{u \in \mathfrak{M} / \mathfrak{M}^{2} \mid u^{2}=0\right\} \subseteq \operatorname{gr}\left(A_{n, 2,2, n+7}^{t}\right)
$$

If $u:=\sum_{i=1}^{n} \lambda_{i} \bar{x}_{i}$ (here $\bar{x}_{i}$ is the class of $x_{i}$ inside of $\mathfrak{M} / \mathfrak{M}^{2}$ ), then $u^{2}:=\lambda_{1}^{2} \bar{x}_{1}^{2}+2 \lambda_{1} \lambda_{2} \bar{x}_{1} \bar{x}_{2}+\lambda_{2}^{2} \bar{x}_{2}^{2}$. Thus $U_{1}=\left\langle\bar{x}_{2}, \ldots, \bar{x}_{n}\right\rangle$ and $U_{2}=\left\langle\bar{x}_{3}, \ldots, \bar{x}_{n}\right\rangle$. It follows that $A_{n, 2,2, n+7}^{1} ¥ A_{n, 2,2, n+7}^{2}$.

Assume now that $H_{A}=(1, n, 3,2,1)$ where $n \geqslant 3$. Decomposition (4) of $H_{A}$ is one of the following:

$$
\begin{aligned}
& (1,2,3,2,1)+(0,0,0,0,0)+(0, n-2,0,0,0), \\
& (1,2,2,2,1)+(0,1,1,0,0)+(0, n-3,0,0,0) .
\end{aligned}
$$

In both cases the algebra $G(A)$ has been described in [5].

The following theorem gives a complete classification up to isomorphisms of the algebras we are interested in. First we fix some notation. Let $u$ and $v$ be acting on $k \backslash\{0, \pm 1, \pm 3\}$ as follows: $u(\alpha)=-\alpha$ and $v(\alpha)=\frac{\alpha-3}{\alpha+1}$. The transformations $u$ and $v$ generate a group $G \cong \mathfrak{S}_{3}$ and the $G$-orbit of $\alpha \in k \backslash\{0, \pm 1, \pm 3\}$ is $G_{\alpha}:=\left\{ \pm \alpha, \pm \frac{\alpha-3}{\alpha+1}, \pm \frac{\alpha+3}{\alpha-1}\right\}$.

Theorem 5.2. Let $n \geqslant 3$ be an integral number. If $A$ is a local, Artinian, Gorenstein, $k$-algebra with $H_{A}=$ $(1, n, 3,2,1)$, then $A \cong A_{n, 3,2, n+7}^{t, \alpha}:=S[n] / J_{t, \alpha}, t=1, \ldots, 7$, where

$$
\begin{aligned}
J_{1,0} & :=\left(x_{1} x_{2}-x_{3}^{2}, x_{1} x_{3}-x_{2}^{3}, x_{2} x_{3}-x_{1}^{3}, x_{1}^{4}-x_{2}^{4}, x_{3}^{3}-x_{1}^{4}, x_{i} x_{j}, x_{j}^{2}-x_{1}^{4}, x_{1}^{5}\right)_{1 \leqslant i<j \leqslant n, j \geqslant 4, i \neq j}, \\
J_{2,0} & :=\left(x_{1} x_{2}, x_{1} x_{3}, x_{2} x_{3}, x_{1}^{4}-x_{2}^{4}, x_{3}^{3}-x_{1}^{4}, x_{i} x_{j}, x_{j}^{2}-x_{1}^{4}\right)_{i \geqslant 1, j \geqslant 4, i \neq j}, \\
J_{3,0} & :=\left(x_{2}^{2}-x_{1}^{3}, x_{1} x_{3}, x_{2} x_{3}, x_{1}^{4}-x_{1}^{3} x_{2}, x_{3}^{3}-x_{1}^{4}, x_{i} x_{j}, x_{j}^{2}-x_{1}^{4}\right)_{i \geqslant 1, j \geqslant 4, i \neq j}, \\
J_{4,0} & :=\left(x_{2}^{2}, x_{1} x_{3}, x_{2} x_{3}, x_{1}^{4}-x_{1}^{3} x_{2}, x_{3}^{3}-x_{1}^{4}, x_{i} x_{j}, x_{j}^{2}-x_{1}^{4}\right)_{i \geqslant 1, j \geqslant 4, i \neq j}, \\
J_{5,0} & :=\left(x_{1}^{3}, x_{2}^{3}, x_{i} x_{j}, x_{j}^{2}-x_{1}^{2} x_{2}^{2}\right)_{i \geqslant 1, j \geqslant 3, i \neq j}, \\
J_{6,0} & :=\left(x_{1}^{3}, x_{2}^{3}-x_{1}^{2} x_{2}, x_{i} x_{j}, x_{j}^{2}-x_{1}^{2} x_{2}^{2}\right)_{i \geqslant 1, j \geqslant 3, i \neq j}, \\
J_{7, \alpha} & :=\left(x_{1}^{3}-\alpha x_{1} x_{2}^{2}, x_{2}^{3}-\alpha x_{1}^{2} x_{2}, x_{i} x_{j}, x_{j}^{2}-x_{1}^{2} x_{2}^{2}\right)_{i \geqslant 1, j \geqslant 3, i \neq j}, \quad \alpha \in k \backslash\{0, \pm 1, \pm 3\} .
\end{aligned}
$$

Moreover, $A_{n, 3,2, n+7}^{t, \alpha} \cong A_{n^{\prime}, 3,2, n^{\prime}+7}^{t^{\prime}, \alpha^{\prime}}$ if and only if $n=n^{\prime}, t=t^{\prime}$ and, in the case $t=t^{\prime}=7, \alpha^{\prime} \in G_{\alpha}$.

Proof. Let $H_{A}=(1,2,3,2,1)+(0,0,0,0,0)+(0, n-2,0,0,0)$. In this case $A \cong S[n] / \operatorname{Ann}(F)$ where $F=G+\sum_{j=3}^{n} y_{j}^{2}$ where $G \in P[2]$ (see Theorem 3.1). Moreover, Equality (6) again implies that both

$$
\operatorname{Ann}(F)=\operatorname{Ann}(G) S[n]+\left(x_{i} x_{j}, x_{j}^{2}-\sigma\right)_{i \geqslant 1, j \geqslant 3, i \neq j}
$$


where $\sigma \circ G=1$ and the Hilbert function of $S[2] / \operatorname{Ann}(G)$ is $(1,2,3,2,1)$. Thanks to the results proved in Section 3 of [5] we know that we can find an automorphism of $S[2]$ such that $\operatorname{Ann}(G)$ can be put in one of the following forms

$$
I_{5,0}:=\left(x_{1}^{3}, x_{2}^{3}\right), \quad I_{6,0}:=\left(x_{1}^{3}, x_{2}^{3}-x_{1}^{2} x_{2}\right), \quad I_{7, \alpha}:=\left(x_{1}^{3}-\alpha x_{1} x_{2}^{2}, x_{2}^{3}-\alpha x_{1}^{2} x_{2}\right),
$$

where $\alpha \in k \backslash\{0, \pm 1, \pm 3\}$. It is easy to check that these three ideals correspond to the polynomials $6 y_{1}^{2} y_{2}^{2}$, $6 y_{1}^{2} y_{2}^{2}+y_{2}^{4}, 6 y_{1}^{2} y_{2}^{2}+\alpha\left(y_{1}^{4}+y_{2}^{4}\right)$ respectively. Thus we can choose $\sigma:=x_{1}^{2} x_{2}^{2} / 24$. The transformation $x_{j} \mapsto$ $x_{j} / \sqrt{4 !}, j \geqslant 3$, finally yields to the ideals $J_{5,0}, J_{6,0}, J_{7, \alpha}$ respectively.

Now we focus our attention on the other case $H_{A}=(1,2,2,2,1)+(0,1,1,0,0)+(0, n-3,0,0,0)$. Again from Theorem 3.1 we know that $A S \boxplus] / \operatorname{Ann}(F)$ where $F=G+{ }_{j=4}^{n} \widehat{y}_{j}^{2}$ where $G$ is a quartic polynomial in $P[3]$. If $G_{4}$ is the leading form of $G, G(A) \cong S[2] / \operatorname{Ann}\left(G_{4}\right)$ has Hilbert function $(1,2,2,2,1)$. Thus [5] (see Section 4) yields that $\operatorname{Ann}\left(G_{4}\right)$ can be put in one of the following forms

$$
I_{1}:=\left(x_{1} x_{2}, x_{1}^{4}-x_{2}^{4}\right), \quad I_{2}:=\left(x_{2}^{2}, x_{1}^{4}-x_{1}^{3} x_{2}\right) .
$$

Correspondingly $G_{4}$ is either $y_{1}{ }^{4}+y_{2}{ }^{4}$ or $y_{1}{ }^{4}+4 y_{1}{ }^{3} y_{2}$. Since $\operatorname{deg}(G)=4$, it follows that either $x^{4}{ }_{1}-x^{4}{ }_{2}$ or $x^{4}{ }_{1}-$ $x^{3}{ }_{1} x_{2}$ is in $\operatorname{Ann}(G)$ respectively. Again Equality (6) implies that $\operatorname{Ann}(F)=\operatorname{Ann}(G) S[n]+\left(x_{i} x\right.$
where the Hilbert function of $S[2] / \operatorname{Ann}(G) \operatorname{is}(1,3,3,2,1)$.

$$
\begin{aligned}
& \left.\operatorname{Ann}(F)=\operatorname{Ann}(G) S[n]+\left(x_{i} x_{j}, x_{j}{ }^{2}-x^{4}\right)_{1}\right)_{i \geqslant 1, j \geqslant 4, i \neq j} \\
& S[2] / \operatorname{Ann}(G) \operatorname{is}(1,3,3,2,1) .
\end{aligned}
$$

We examine the second case, the first one being similar. Since $G(A)$ is a quotient $\operatorname{of} \operatorname{gr}(A)$, it follows that the classes of $x_{1}, x_{2}, x^{2}{ }_{1}, x^{2}{ }_{2}, x^{3}{ }_{1}, x^{3}{ }_{2}$ and $x^{4}{ }_{1}$ in $G(A)$ can be lifted to generators of $\bigoplus_{i} \mathfrak{M}^{i} / \mathfrak{M}^{i+1} \subseteq \operatorname{gr}(A)$ as $k$-vector space, thus of $\mathfrak{M}^{2} \subseteq A$, due to Nakayama's Lemma. In particular, $x^{2}{ }_{1}, x^{2}{ }_{2}, x^{3}{ }_{1}, x^{3}{ }_{2}, x^{4}{ }_{1}$ are linearly independent modulo $\operatorname{Ann}(G)$. In what follows we will denote by $a_{i}$ the class of $x_{i}$ in $S[3] / \operatorname{Ann}(G)$.

Notice that $H_{S[3] / \operatorname{Ann}(G)}(2)=3$, thus, besides $a^{2}{ }_{1}, a_{2}^{2}$, there exists another generator in degree 2 . We can always assume that such a generator is $a_{i} a_{3}$. Via a transformation of the form $a_{3} \mapsto a_{i}+a_{3}$ we can finally choose $a_{1}, a_{2}, a_{3}, a^{2}{ }_{1}, a_{2}^{2}, a^{2}{ }_{3}, a^{3}{ }_{1}, a^{3}{ }_{2}, a^{4}{ }_{1}$ as a basis of the ideal generated by $\left(a_{1}, a_{2}, a_{3}\right)$ as $k$-vector space. Recall that $a_{1}^{4}=a_{2}^{4}$ in $A$.

There are quadratic forms $q_{i, j} \in\left(a_{1}^{2}, a_{2}^{2}, a_{3}^{2}\right), 1 \leqslant i<j \leqslant 3$, such that $a_{i} a_{j}-q_{i, j} \in\left(a_{1}, a_{2}, a_{3}\right)^{3}$. Thus $a_{i} a_{j}-q_{i, j} \in \operatorname{ldf}(\operatorname{Ann}(G))=\operatorname{Ann}(\operatorname{tdf}(G))$. Since $y_{1}^{3}, y_{2}^{3} \in \operatorname{tdf}(G)$, it follows that $q_{i, j}=u_{i, j} a_{3}^{2}$ where $u_{i, j} \in k$. Thus

$$
a_{i} a_{j}=u_{i, j} a_{3}^{2}+v_{i, j}^{1} a_{1}^{3}+v_{i, j}^{2} a_{2}^{3}+w_{i, j} a_{1}^{4}
$$

for suitable $u_{i, j}, v_{i, j}^{1}, v_{i, j}^{2}, w_{i, j} \in k$.

The linear automorphism of $S[3] / \operatorname{Ann}(G)$ given by $b_{3}:=a_{3}, b_{i}:=a_{1}+u_{i, 3} a_{3}, i=1$, 2 , allows us to assume $u_{i, 3}=0$ in Eq. (9), $i=1,2$. The automorphism defined by $c_{1}:=b_{1}+v_{1,2}^{2} b_{2}^{2}, c_{2}:=b_{2}+v_{1,2}^{1} b_{1}^{2}, c_{3}:=b_{3}$ allows us to assume $v_{1,2}^{1}=v_{1,2}^{2}=0$. Finally, the automorphism $\left(d_{1}, d_{2}, d_{3}\right):=\left(c_{1}, c_{2}+w_{1,2} c_{1}^{3}, c_{3}\right)$ allows us to assume also $w_{1,2}=0$, i.e.

$$
d_{1} d_{2}=u_{1,2} d_{3}^{2}
$$

Notice that, after the above automorphisms, $d_{1}, d_{2}, d_{3}, d_{1}^{2}, d_{2}^{2}, d_{3}^{2}, d_{1}^{3}, d_{2}^{3}, d_{1}^{4}$ still is a basis of the ideal $\left(d_{1}, d_{2}, d_{3}\right)=\left(a_{1}, a_{2}, a_{3}\right)$ as $k$-vector space.

Since $u_{i, 3}=0$, then Eq. (9) and the vanishing $\left(d_{1}, d_{2}, d_{3}\right)^{5}=0$ yield $d_{i}^{3} d_{3}=0, \quad i=1,2$.

Substituting in Eq. (9) we also obtain $d_{i} d_{3}^{2}=0, \quad i=1,2$. Consequently Eq. (10) yield $d_{1}^{2} d_{2}=d_{1} d_{2}^{2}=0$. 
Let $d_{3}^{3}=\lambda_{1} d_{1}^{3}+\lambda_{2} d_{2}^{3}+\lambda_{1}^{4}=0, \quad \lambda_{1}, \lambda_{2}, \lambda \in k$. The conditions $d_{i}^{3} d_{3}=0$ above also imply $d_{3}^{4}=0 . \quad$ It is easy to check that $a_{i}=d_{i}-u_{i, 3} d_{3}$ modulo $\left(d_{1}, d_{2}, d_{3}\right)^{5}, i=1,2$, thus the conditions $a_{1}^{4}=a_{2}^{4}$ and $d_{3}^{4}=0$ finally give $d_{1}^{4}=d_{2}^{4}$.

We have $d_{i} d_{3}^{3}=\lambda_{i} d_{i}^{4}$, hence $\lambda_{i}=0$ because $d_{i}^{4} \neq 0, \quad i=1$, 2. If $\lambda=0$ too, then Eq. (9) would imply $d_{1} d_{3}^{2}=$ $d_{2} d_{3}^{2}=d_{3}^{3}=0$, hence $d_{3}^{2} \in \operatorname{Soc}(S[3] / \operatorname{Ann}(G))$. But this is not possible because $d_{3}^{2}$ is a minimal generator of $\left(d_{1}, d_{2}, d_{3}\right)^{2}$. Thus we can assume $\lambda=1$, whence $d_{3}^{3}=d_{1}^{4}$.

Let $\left(e_{1}, e_{2}, e_{3}\right):=\left(d_{1}, d_{2}, d_{3}+v_{1,3}^{1} d_{1}^{2}+v_{2,3}^{2} d_{2}^{2}+w_{1,3} d_{1}^{3}+w_{2,3} d_{2}^{3}\right)$. It is easy to check that we still have $e_{1} e_{3}^{2}=0, \quad i=1,2$, and

$$
e_{3}^{3}=e_{1}^{4}, \quad e_{1}^{4}=e_{2}^{4}
$$

Then $e_{1} e_{3}=v_{1,3}^{2} e_{2}^{3}+v_{2,3}^{2} e_{1} e_{2}^{2}+w_{2,3} e_{1} e_{2}^{3}=v_{1,3}^{2} e_{2}^{3}$ because we checked above that $e_{1} e_{2}^{2}=0$. Similarly $e_{2} e_{3}=v_{2,3}^{1} e_{1}^{3}$.

We have $v_{1,3}^{2} e_{2}^{4}=e_{2}\left(e_{1} e_{3}^{\prime}\right)=e_{1}\left(e_{2} e_{3}\right)=v_{2,3}^{1} e_{1}^{4}$, thus $v_{1,3}^{2}=v_{2,3}^{1}$. Due to Equalities (10), (11) and to $e_{i} e_{3}^{2}=0, \quad i=1,2$, we obtain $u_{1,2}=v_{1,3}^{2}=v_{2,3}^{1}$ because $e_{1} e_{2} e_{3}=v_{1,3}^{2} e_{2}^{4}=v_{2,3}^{1} e_{1}^{4}=u_{1,2} e_{3}^{3}$.

Either $u_{1,2}=v_{1,3}^{2}=v_{2,3}^{1}=0 \quad$ or $\quad u_{1,2}=v_{1,3}^{2}=v_{2,3}^{1}=\mu^{2}$ for a suitable non-zero $\mu \in k$. In the former case we recover $J_{2,0}$. In the latter the transformation $\left(f_{1}, f_{2}, f_{3}\right):=\left(\mu^{3} e_{1}, \mu^{3} e_{2}, \mu^{4} e_{3}\right)$ allows us to assume $u_{1,2}=v_{1,3}^{2}=$ $v_{2,3}^{1}=1$. In this case we thus recover $J_{1,0}$.

In order to complete the proof we have to show that $A_{n, 3,2, n+7}^{t, \alpha} \cong A_{n^{\prime}, 3,2, n^{\prime}+7}^{t^{\prime}, \alpha^{\prime}}$ if and only if $n=n^{\prime}$, $t=t^{\prime}$ and, in the case $t=t^{\prime}=7, \alpha^{\prime} \in G_{\alpha}$, one implication being an easy check. Conversely, if $A_{n, 3,2, n+7}^{t, \alpha} \cong A_{n^{\prime}, 3,2, n^{\prime}+7}^{t^{\prime}, \prime^{\prime}}$, then $n=n^{\prime}$. Let $H_{t, \alpha}:=\operatorname{ldf}\left(J_{t, \alpha}\right)+\left(x_{3}, \ldots, x_{n}\right) \subseteq S[n]$. There exists an epimorphism $\operatorname{gr}\left(A_{n, 3,2, n+7}^{t, \alpha}\right) \rightarrow S[n] / H_{t, \alpha}$.

Moreover (see Sections 3 and 4 of [5] for the following results and undefined notation), we also have that $S[n] /$ $H_{t, \alpha} \cong A_{2,2,2,8}^{1}$ for $t \in\{1,2\}, S[n] / H_{4,0} \cong A_{2,2,2,8}^{2}$ for $t \in\{3,4\}$ and $S[n] / H_{t, \alpha} \cong A_{2,3,2,9}^{8-t, \alpha}$ for

$t \in\{5,6,7\}$ (see Section 4 of [5]). Since both $A_{2,3,2,9}^{t, \alpha}$ and $A_{2,2,2,8}^{t}$ are Artinian Gorenstein local $k$-algebras with socle degree 4 , it follows that

$$
G\left(A_{n, 3,2, n+7}^{t, \alpha}\right) \cong \begin{cases}A_{2,2,2,8}^{2} & \text { if } t=1,2, \\ A_{2,2,2,8}^{1} & \text { if } t=3,4 \\ A_{2,3,2,9}^{8-t, \alpha} & \text { if } t=5,6,7 .\end{cases}
$$

In Section 3 of [5] we proved that $A_{2,3,2,9}^{8-t, \alpha} \alpha^{\prime} \cong A_{2,3,2,9}^{8-t^{\prime}, \alpha^{\prime}}$ if and only if $t=t^{\prime}$ and, in the case $t=t^{\prime}=1$, also $\in G_{\alpha}$.

It remains to prove that $A_{n, 3,2, n+7}^{1,0} ¥ A_{n, 3,2, n+7}^{2,0}$ and $A_{n, 3,2, n+7}^{3,0} ¥ A_{n, 3,2, n+7}^{4,0}$. Let us first examine the second case. Consider the set

$$
U_{t}:=\left\{u \in A_{n, 3,2, n+7}^{t, 0} \mid u^{2} \in \mathfrak{M}^{4}\right\} .
$$

Let $\bar{x}_{i}$ be the class of $x_{i}$ inside $S[n] / J_{t}$. If

$$
u:=\sum_{i=1}^{n} \lambda_{i} \bar{x}_{i}+\lambda_{n+1} \bar{x}_{1}^{2}+\lambda_{n+2} \bar{x}_{1} \bar{x}_{2}+\lambda_{n+3} \bar{x}_{1}^{3}+\lambda_{n+4} \bar{x}_{1}^{2} \bar{x}_{2}+\lambda_{n+5} \bar{x}_{1}^{4},
$$

then

$$
\begin{aligned}
u^{2}= & \lambda_{1}^{2} \bar{x}_{1}^{2}+2 \lambda_{1} \lambda_{2} \bar{x}_{1} \bar{x}_{2}+\lambda_{2}^{2} \bar{x}_{2}^{2}+\lambda_{3}^{2} \bar{x}_{3}^{2}+2 \lambda_{1} \lambda_{n+1} \bar{x}_{1}^{3}+\left(2 \lambda_{2} \lambda_{n+1}+2 \lambda_{1} \lambda_{n+2}\right) \bar{x}_{1}^{2} \bar{x}_{2} \\
& +\left(\sum_{i=4}^{n+1} \lambda_{i}^{2}+2 \lambda_{1} \lambda_{n+3}+2 \lambda_{1} \lambda_{n+4}+2 \lambda_{2} \lambda_{n+2}+2 \lambda_{2} \lambda_{n+3}+2 \lambda_{n+1} \lambda_{n+2}\right) \bar{x}_{1}^{4} .
\end{aligned}
$$


If $u \in U_{t}$ we must have $\lambda_{1}=\lambda_{3}=\lambda_{2} \lambda_{n+1}=0$. Moreover, $u \in U_{3}$ if and only if $\lambda_{2}=0$ too. It follows that

$$
\begin{aligned}
U_{3} & =\left\langle\bar{x}_{4}, \ldots, \bar{x}_{n}, \bar{x}_{1}^{2}, \bar{x}_{1} \bar{x}_{2}, \bar{x}_{1}^{3}, \bar{x}_{1}^{2} \bar{x}_{2}, \bar{x}_{1}^{4}\right\rangle, \\
U_{4} & =\left\langle\bar{x}_{2}, \bar{x}_{4}, \ldots, \bar{x}_{n}, \bar{x}_{1} \bar{x}_{2}, \bar{x}_{1}^{3}, \bar{x}_{1}^{2} \bar{x}_{2}, \bar{x}_{1}^{4}\right\rangle \cup\left\langle\bar{x}_{4}, \ldots, \bar{x}_{n}, \bar{x}_{1}^{2}, \bar{x}_{1} \bar{x}_{2}, \bar{x}_{1}^{3}, \bar{x}_{1}^{2} \bar{x}_{2}, \bar{x}_{1}^{4}\right\rangle,
\end{aligned}
$$

hence $A_{n, 2,2, n+7}^{3} \not A_{n, 2,2, n+7}^{4}$.

Finally, we will prove that $A_{n, 3,2, n+7}^{1,0} ¥ A_{n, 3,2, n+7}^{2,0}$ by checking that $\operatorname{gr}\left(A_{n, 3,2, n+7}^{1,0}\right) \varsubsetneqq \operatorname{gr}\left(A_{n, 3,2, n+7}^{2,0}\right)$. Recall that, if $R$ is a regular local ring with maximal ideal $\mathfrak{M}$ and $A:=R / I$ for a suitable ideal $I \subseteq \mathfrak{M}^{2}$, then the minimal number of generators $\mu(I)$ of $I$ depends only on $A$ since it coincides with the first deviation of $A$ (see [22], Section 21: in particular, see Theorem 21.1(iii)).

Recall that $\operatorname{gr}\left(A_{n, 3,2, n+7}^{t, 0}\right) \cong S[n] / \operatorname{ldf}\left(J_{t, 0}\right), t=1,2$ (see Identity $\left.(3)\right)$. Trivially

$$
\begin{aligned}
& \operatorname{ldf}\left(J_{1,0}\right)=\left(x_{1} x_{2}-x_{3}^{2}, x_{1} x_{3}, x_{2} x_{3}, x_{1}^{4}-x_{2}^{4}, x_{3}^{3}, x_{i} x_{j}, x_{j}^{2}\right)_{i \geqslant 1, j \geqslant 4, i \neq j}, \\
& \operatorname{ldf}\left(J_{2,0}\right)=\left(x_{1} x_{2}, x_{1} x_{3}, x_{2} x_{3}, x_{1}^{4}-x_{2}^{4}, x_{3}^{3}, x_{i} x_{j}, x_{j}^{2}\right)_{i \geqslant 1, j \geqslant 4, i \neq j} .
\end{aligned}
$$

It is not difficult to check that the system of generators for $\operatorname{ldf}\left(J_{2,0}\right)$ indicated above is minimal and that $x_{3}^{3}=-x_{3}\left(x_{1} x_{2}-x_{3}^{2}\right)+x_{2}\left(x_{1} x_{3}\right)$ holds in $\operatorname{ldf}\left(J_{1,0}\right) . \operatorname{Thus} \mu\left(\operatorname{ldf}\left(J_{1,0}\right)\right)=\mu\left(\operatorname{ldf}\left(J_{2,0}\right)\right)-1<\mu\left(\operatorname{ldf}\left(J_{2,0}\right)\right)$, whence $A_{n, 3,2, n+7}^{1,0} \nsucceq A_{n, 3,2, n+7}^{2,0}$.

\section{On the singular locus of $\mathcal{H} i b_{11}^{G}\left(\mathbb{P}_{k}^{N}\right)$}

In the previous papers [8] and [9], we gave a complete description of the singular locus of $\mathcal{H} i l b_{d}^{G}\left(\mathbb{P}_{k}^{N}\right)$ for $d \leqslant 10$. In the present section we prove partial results about $\operatorname{Sing}\left(\mathcal{H i l b} b_{11}^{G}\left(\mathbb{P}_{k}^{N}\right)\right)$, along the same lines. Since $\mathcal{H i l b} b_{d}^{G}\left(\mathbb{P}_{k}^{N}\right)=\mathcal{H i l b}{ }_{d}^{G, g e n}\left(\mathbb{P}_{k}^{N}\right)$ for $d \leqslant 11$, it suffices to check whether $h^{0}\left(X, \mathcal{N}_{X}\right)>d N=\operatorname{dim}\left(\mathcal{H} i b_{d}^{G}\left(\mathbb{P}_{k}^{N}\right)\right)$, $\mathcal{N}_{X}$ being the normal sheaf of $X \subseteq \mathbb{P}_{k}^{N}$.

As pointed out in the introduction, $X \in \operatorname{Sing}\left(\mathcal{H} i l b_{d}^{G}\left(\mathbb{P}_{k}^{N}\right)\right)$ if and only if there exists an irreducible component $Y \subseteq X$ which is singular in the corresponding Hilbert scheme. Moreover, in Lemma 2.3 of [8] it is proved that such a dimension can be computed with respect to any embedding, as for its smoothability. Thus it makes sense to say that the algebra $A$ is unobstructed if and only if the same is true for some embedding $\operatorname{spec}(A) \subseteq \mathbb{P}_{k}^{N}$

Moreover, we can also restrict our attention to the case $n \geqslant 4$, since Gorenstein algebras $A$ with $H_{A}(1) \leqslant 3$ are automatically unobstructed (see e.g. Proposition 2.5 and the references therein).

In particular, all the schemes in $\mathcal{H} i b_{11}^{G}\left(\mathbb{P}_{k}^{N}\right)$ containing as a connected component one of the obstructed

\begin{tabular}{|c|c|c|c|}
\hline Hilbert function & Notation & Obstructedness & Reference \\
\hline$(1, n, 1, \ldots, 1)$ & $A_{n, d}$ & iff $n \geqslant 4$ & [8], Section 5 \\
\hline$(1, n, 2,1, \ldots, 1)$ & $A_{n, 2, d}^{t}$ & iff $n \geqslant 4$ & [8], Section 5 \\
\hline$(1,4,2,2,1)$ & $A_{4,2,2,10}^{t, 2, a}$ & for all $t$ & {$[8]$, Section 5} \\
\hline$(1,4,2,2,1,1)$ & $A_{4,2,2,11}^{t}$ & for all $t$ & Proposition 6.1 below \\
\hline$(1,4,3,1)$ & $A_{4,3,9}^{t, 2,11}$ & iff $t=4,5,6$ & {$[8]$, Section 5} \\
\hline$(1,4,3,1,1)$ & $A_{4,3,10}^{t, 3}$ & iff $t=2,3,4,5,6$ & [8], Theorem 4.1 \\
\hline$(1,4,3,1,1,1)$ & $A_{4,3,11}^{t, 3,10}$ & iff $t=2,3,4,5,6$ & Proposition 6.1 below \\
\hline$(1,4,3,2,1)$ & $A_{4,3,2,11}^{t, \alpha, 11}$ & iff $t=2,3,4,5,6,7$ & Proposition 6.3 below \\
\hline$(1,5,2,2,1)$ & $A_{5,2,11}^{t}, 3,2,11$ & for all $t$ & Proposition 6.1 below \\
\hline$(1,5,3,1)$ & $A_{5,3,10}^{t, 2,2,11}$ & for allt & {$[8]$, Section 5} \\
\hline$(1,5,3,1,1)$ & $A_{5}^{t}, 3,10$ & for all $t$ & Proposition 6.1 below \\
\hline$(1,6,3,1)$ & $A_{6,3,11}^{t, 3,11}$ & for all $t$ & Proposition 6.2 below \\
\hline$(1,4,4,1)$ & $\begin{array}{l}A_{4,4,10} \\
A_{4,11}\end{array}$ & iff $\beta=3$ & [9], Theorem $4.7[10]$ \\
\hline$(1,4,4,1,1)$ & $A_{4,4,11}$ & sometimes & below Proposition 6.2 \\
\hline$(1,5,4,1)$ & $A_{5,4,11}$ & every such an algebra & below \\
\hline
\end{tabular}
irreducible schemes $\operatorname{spec}(A)$ described in Theorems 4.1 and 4.7 of [9], are obstructed too.

We quickly list the obstructed algebras up to dimension 11, using the results proven both in the two aforementioned theorems and in the remaining part of the present section. For the notation see also [9]. 
Thus the cases that we have to investigate are

$$
\begin{array}{rrr}
(1,4,2,2,1,1), \quad(1,4,3,1,1,1), \quad(1,4,3,2,1), & (1,4,4,1,1), \\
(1,5,2,2,1), \quad(1,5,3,1,1), & (1,5,4,1), & (1,6,3,1) .
\end{array}
$$

In all the aforementioned cases but $H_{A}=(1,4,4,1,1)$, we are able to give a simple answer.

In Theorem 5.1 we gave a complete classification of algebras $A$ with $H_{A}=(1,4,2,2,1,1)$. Let $X:=\operatorname{spec}(A)$ : using Formula (8) and any computer algebra software (e.g. we used CoCoA: see [11]), one checks via direct computation that $h^{0}\left(X, \mathcal{N}_{X}\right)=49>44=\operatorname{dim}\left(\mathcal{H} i l b_{11}^{G}\left(\mathbb{P}_{k}^{4}\right)\right)$.

The classification of algebras $A$ such that $H_{A}=(1, n, 3,1, \ldots, 1)$ is described in [5], Section 6 . We have seven isomorphism classes denoted by $A_{n, 3,11}^{t}, t=0, \ldots, 6$. We can again make a direct computation using Formula (8) as in the previous case, obtaining

$$
h^{0}\left(X, \mathcal{N}_{X}\right)= \begin{cases}44 & \text { if } n=4 \text { and } t=0,1, \\ 49 & \text { if } n=4 \text { and } t=2, \ldots, 6 \\ 62 & \text { if } n=5 \text { and } t=0,1 \\ 69 & \text { if } n=5 \text { and } t=2, \ldots, 6\end{cases}
$$

The classification of algebras $A$ such that $H_{A}=(1,5,2,2,1)$ is described in [5], Section 4 . We have three non-isomorphic algebras denoted by $A_{5,2,2,11}^{t}, t=1,2,3$. Again a direct computation as in the previous cases yields $h^{0}\left(X, \mathcal{N}_{X}\right)=69$ in all the three cases.

We summarize the above results in the following

Proposition 6.1. Let $A$ be a local, Artinian, Gorenstein k-algebra such that $H_{A}$ is either $(1,4,2,2,1,1)$, or $(1,5$, $2,2,1)$, or $(1,4,3,1,1,1)$, or $(1,5,3,1,1)$. Then $A$ is obstructed but the case $A \cong A_{4,3,11}^{t}$ with $t=0,1$.

Now consider the case of an Artinian Gorenstein local algebra $A$ with $H_{A}$ equal to either $(1,6,3,1)$, or $(1,5,4,1)$. We have the following, more general, result.

Proposition 6.2. Let $A$ be a local, Artinian, Gorenstein $k$-algebra such that $H_{A}=(1, n, m, 1)$ with $n \geqslant 5$ and $m=3,4$. Then $A$ is obstructed.

Proof. Assume that the statement is true when $n=5$ and we prove it for $n>5$ by induction on $n$. In Proposition 4.8 of [8] and Theorem 4.2 of [6] flat families $\mathcal{A} \rightarrow \mathbb{A}_{k}^{1}$ with special fibre $A$ and general fibre isomorphic to $A^{\prime} \oplus k$ with $H_{A^{\prime}}=(1, n-1, m, 1)$ are explicitly constructed. Correspondingly, we have a flat family of schemes $\mathcal{X} \rightarrow \mathbb{A}_{k}^{1}$ in $\mathcal{H} i l b_{d}^{G}\left(\mathbb{P}_{k}^{n}\right)$. The general fibre of $\mathcal{X}$ is obstructed since it contains $\operatorname{spec}\left(A^{\prime}\right)$ which is obstructed by induction hypothesis. It follows that the limit point $X$ is obstructed too.

Now we prove the statement for $n=5$. When $m=3$ the statement is part of Theorem 4.1 of [9]. Thus it remains to examine the case $m=4$. We have an isomorphism $A \cong S[5] / J$, hence an embedding $X \subseteq \mathbb{A}_{k}^{5}$. We will check that $h^{0}\left(X, \mathcal{N}_{X}\right)>55$. Using Formula (8) above, it suffices to verify that

$$
\operatorname{dim}_{k}\left(S[5] / J^{2}\right)>66 .
$$

Since $\operatorname{dim}_{k}\left(S[5] / J^{2}\right)=\operatorname{dim}_{k}\left(\operatorname{gr}\left(S[5] / J^{2}\right)\right)=\operatorname{dim}_{k}\left(S[5] / \operatorname{ldf}\left(J^{2}\right)\right)$ (see Formula (1)), we can finally reduce to check that

$$
\operatorname{dim}_{k}\left(S[5] / \operatorname{ldf}\left(J^{2}\right)\right)>66 .
$$

We now deal with the generators of $J^{2}$. A system of generators for $J^{2}$ certainly contains the products of the elements of a system of generators of $J$, thus we first describe them. In Theorem 4.1 of [12] it is 
proved that, up to a proper choice of the variables $x_{1}, \ldots, x_{5}$, there is a cubic form $G \in P[4]$ such that $J=\operatorname{Ann}\left(G+y_{5}^{2}\right)$.

In particular we can apply Eq. (5) to such an ideal $J$ obtaining

$$
J=\operatorname{Ann}(G) S[4]+\left(x_{5}^{2}-2 \sigma, x_{i} x_{5}\right)_{1 \leqslant i \leqslant 4}
$$

for a suitable cubic form $\sigma \in S[4]$. We know that $\operatorname{Ann}(G) \subseteq S[4]$ is generated by six quadratic forms $q_{1}, \ldots, q_{6}$ and, possibly, by other cubic forms (see [9], Lemma 4.2). Thus $J^{2}$ is also generated by the products

$$
q_{i} q_{i^{\prime}}, \quad q_{i} x_{j} x_{5}, \quad q_{i}\left(x_{5}^{2}-2 \sigma\right), \quad x_{j} x_{j^{\prime}} x_{5}^{2}, \quad x_{j} x_{5}\left(x_{5}^{2}-2 \sigma\right), \quad\left(x_{5}^{2}-2 \sigma\right)^{2},
$$

$i=1, \ldots, 6, j, j^{\prime}=1, \ldots, 4$, and, possibly, by other polynomials whose lower degree forms have degree at least five. Thus, in degree up to 3 , the ideal $\operatorname{ldf}\left(J^{2}\right)$ is zero and in degree 4 it is generated by the lower degree forms of the generators listed above, i.e.

$$
q_{i} q_{i^{\prime}}, \quad q_{i} x_{j} x_{5}, \quad q_{i} x_{5}^{2}, \quad x_{j} x_{j^{\prime}} x_{5}^{2}, \quad x_{j} x_{5}^{3}, \quad x_{5}^{4},
$$

$i, i^{\prime}=1, \ldots, 6, j, j^{\prime}=1, \ldots, 4, j \neq j^{\prime}$. Let $W$ be the $k$-space generated by the above quartic forms.

The dimension of the subspace in $S[5]$ of polynomials of degree up to 4 is 126 , thus the first member of Inequality (12) is at least $126-\operatorname{dim}_{k}(W)$. We have to find an upper bound for $\operatorname{dim}_{k}(W)$.

The forms $q_{i} q_{i^{\prime}}$ are at most 21. The forms $q_{i} x_{j}$ are cubic forms in $\operatorname{Ann}(G)$. Since the Hilbert function of $S[4] / \operatorname{Ann}(G)$ is $(1,4,4,1)$ they are at most 19. It follows that at most 19 among the quartic forms $q_{i} x_{j} x_{5}$ are linearly independent.

We would have to add the other six forms $q_{i} x_{5}^{2}, i=1, \ldots, 6$. But these forms are contained in the space generated by the forms $x_{j} x_{j^{\prime}} x_{5}^{2}$. The forms $x_{j} x_{j^{\prime}} x_{5}^{2}$ are exactly 10 . It remains only the forms $x_{j} x_{5}^{3}$, which are 4 , and $x_{5}^{4}$.

The above computations show that $\operatorname{dim}_{k}\left(W_{4}\right) \leqslant 55$, thus $\operatorname{dim}_{k}\left(S[5] / \operatorname{ldf}\left(J^{2}\right)\right) \geqslant 126-55=71>66$. Taking into account of Inequality (12), the proof of the statement is now complete.

It remains to examine the case of algebras $A$ with $H_{A}=(1,4,3,2,1)$. In this case we described in Theorem 5.2 a complete classification. We have a 1-dimensional family and six other algebras. These last six cases can be treated as in the proof of Proposition 6.2 obtaining

$$
h^{0}\left(X, \mathcal{N}_{X}\right)= \begin{cases}44 & \text { if } t=1 \\ 49 & \text { if } t=2, \ldots, 6\end{cases}
$$

In the first case, when we have a continuous family, we cannot compute such a dimension directly: we prove the obstructedness with the same argument used in the proof of Proposition 6.2.

Proposition 6.3. Let $A$ be a local, Artinian, Gorenstein k-algebra such that $H_{A}=(1,4,3,2,1)$. Then $A$ is obstructed but the case $A \cong A_{4,3,2,11}^{1}$.

Proof. It remains to examine the case when $X:=\operatorname{spec}(S[4] / J)$ where the ideal $J$ is generated by two cubic forms $q_{1}, q_{2} \in S[2]$ and by $\left(x_{1} x_{3}, x_{2} x_{3}, x_{1} x_{4}, x_{2} x_{4}, x_{3} x_{4}, x_{3}^{2}-x_{1}^{2} x_{2}^{2}, x_{4}^{2}-x_{1}^{2} x_{2}^{2}\right)$ (see Theorem 5.2).

The ideal $J^{2}$ is also generated by the products of the aforementioned polynomials. Again, in degree up to 3 , the ideal $\operatorname{ldf}\left(J^{2}\right)$ is zero. In degree 4 it is generated by the lower degree forms of degree 4 of the aforementioned products. The same is true in degree 5 . Thus $\operatorname{ldf}\left(J^{2}\right)$ has the following generators in degree up to 5 :

$$
q_{i} x_{j} x_{h}, \quad q_{i} x_{h} x_{h^{\prime}}, \quad x_{j} x_{j^{\prime}} x_{h} x_{h^{\prime}}, \quad x_{j} x_{h} x_{h^{\prime}}^{2}, \quad x_{h}^{2} x_{h^{\prime}}^{2},
$$


$i=1,2, j, j^{\prime}=1, \ldots, 4, h, h^{\prime}=3,4, j \neq h, j^{\prime} \neq h^{\prime}$. It is easy to check that there are exactly 22 generators of degree 4 . The generators of degree 5 which do not come from products containing $q_{i}$ are exactly 40 . The forms $q_{i} x_{h} x_{h^{\prime}}$ are all in the space generated by $x_{j} x_{j^{\prime}} x_{h} x_{h^{\prime}}$. Finally, there are at most 8 other generators of the form $q_{i} x_{j} x_{h}$. Thus the generators of $\operatorname{ldf}\left(J^{2}\right)$ up to degree 5 are at most 70 . On the other hand the dimension of the space of polynomials of degree up to 5 in $S[4]$ is 126 . We conclude that

$$
\operatorname{dim}_{k}\left(S[4] / J^{2}\right)=\operatorname{dim}_{k}\left(S[4] / \operatorname{ldf}\left(J^{2}\right)\right) \geqslant 56>55 .
$$

Formula (8) then yields the obstructedness of the scheme $X$.

\section{References}

[1] M.F. Atiyah, I.G. Macdonald, Introduction to Commutative Algebra, Addison-Wesley Publishing Co., 1969.

[2] W. Bruns, J. Herzog, Cohen-Macaulay Rings, II edition, Cambridge University Press, 1998.

[3] W. Buczyńska, J. Buczyński, Secant varieties to high degree Veronese reembeddings, catalecticant matrices and smoothable Gorenstein schemes, J. Algebr. Geom. 23 (2014) 63-90.

[4] D.A. Cartwright, D. Erman, M. Velasco, B. Viray, Hilbert schemes of 8 point in $\mathbb{A}^{d}$, Algebra Number Theory 3 (2009) $763-795$.

[5] G. Casnati, Isomorphism types of Artinian Gorenstein local algebras of multiplicity at most 9, Commun. Algebra 38 (2010) $2738-2761$.

[6] G. Casnati, J. Elias, R. Notari, M.E. Rossi, Poincaré series and deformations of Gorenstein local algebras with low socle degree, Commun. Algebra 41 (2013) 1049-1059.

[7] G. Casnati, J. Jelisiejew, R. Notari, On the rationality of Poincaré series of Gorenstein algebras via Macaulay's correspondence, arXiv:1307.1676 [Math.AC], 5 July 2013.

[8] G. Casnati, R. Notari, On the Gorenstein locus of some punctual Hilbert schemes, J. Pure Appl. Algebra 213 (2009) 2055-2074.

[9] G. Casnati, R. Notari, On the irreducibility and the singularities of the Gorenstein locus of the punctual Hilbert scheme of degree 10, J. Pure Appl. Algebra 215 (2011) 1243-1254.

[10] G. Casnati, R. Notari, A structure theorem for 2-stretched Gorenstein algebras, arXiv:1312.2191 [Math.AC], 8 Dec 2013.

[11] CoCoATeam, CoCoA: a system for doing Computations in Commutative Algebra, available at http://cocoa.dima.unige.it.

[12] J. Elias, M.E. Rossi, Isomorphism classes of short Gorenstein local rings via Macaulay's inverse system, Trans. Am. Math. Soc. 364 (2012) 4589-4604.

[13] J. Elias, G. Valla, Isomorphism classes of certain Artinian Gorenstein algebras, Algebr. Represent. Theory 14 (2011) 429-448.

[14] J. Emsalem, Géométrie des points épais, Bull. Soc. Math. Fr. 106 (1978) 399-416.

[15] J. Emsalem, A. Iarrobino, Réseaux de coniques et algèbres de longueur 7 associées, Université de Paris VII, preprint, 1977.

[16] J. Fogarty, Algebraic families on an algebraic surface, Am. J. Math. 90 (1968) 511-521.

[17] A.V. Geramita, Catalecticant varieties, in: F. van Oystaeyen (Ed.), Commutative Algebra and Algebraic Geometry (Fer-rara), in: Lect. Notes Pure Appl. Math., vol. 206, Dekker, New York, 1999, pp. 143-156.

[18] R. Hartshorne, Algebraic Geometry, G.T.M., vol. 52, Springer, 1977.

[19] A. Iarrobino, Reducibility of the families of 0-dimensional schemes on a variety, Invent. Math. 15 (1972) $72-77$.

[20] A. Iarrobino, Associated graded algebra of a Gorenstein Artin algebra, Mem. Am. Math. Soc. 107 (514) (1994), viii+115 pp.

[21] A. Iarrobino, V. Kanev, Power Sums, Gorenstein Algebras, and Determinantal Loci, Lect. Notes Math., vol. 1721, Springer, 1999.

[22] H. Matsumura, Commutative Ring Theory, Cambridge U.P., 1980.

[23] G. Mazzola, The algebraic and geometric classification of associative algebras of dimension five, Manuscr. Math. 27 (1979) 81-101.

[24] G. Mazzola, Generic finite schemes and Hochschild cocycles, Comment. Math. Helv. 55 (1980) 267-293.

[25] B. Poonen, The moduli space of commutative algebras of finite rank, J. Eur. Math. Soc. 10 (2008) 817-836.

[26] B. Poonen, Isomorphism types of commutative algebras of finite rank over an algebraically closed field, in: Kristin E. Lauter, Kenneth A. Ribet (Eds.), Computational Arithmetic Geometry, in: Contemp. Math., vol. 463, AMS, 2008, pp. 111-120.

[27] C. Raicu, $3 \times 3$ minors of catalecticants, arXiv:1011.1564v3 [math.AG], 8 May 2013.

[28] M. Stoia, Points de Gorenstein d'un morphisme, C. R. Acad. Sci. Paris, Ser. A-B 281 (1975) A847-A849. 